\title{
EFFECTS OF SADNESS AND HOSTILITY ON DEPRESSIVE ATTENTIONAL ALLOCATION PROCESSES
}

\section{BY}

\author{
Melinda A. Gaddy
}

Submitted to the graduate degree program in Clinical Psychology and the Graduate Faculty of the University of Kansas in partial fulfillment of the requirements for the degree of Master's of Arts

Date defended: 
The Thesis Committee for Melinda A. Gaddy certifies that this is the approved Version of the following thesis:

EFFECTS OF SADNESS AND HOSTILITY ON DEPRESSIVE ATTENTIONAL ALLOCATION PROCESSES

Committee Chairperson:

Date approved: 


\section{Acknowledgments}

No words are sufficient to describe my late mother's contribution to my life. I owe every bit of my existence to her, and this thesis is dedicated to her memory.

I would like to express my sincerest gratitude for the invaluable guidance offered by each of the faculty members on my thesis committee, Rick Ingram, Kristopher Preacher, and Stephen Ilardi. I must especially acknowledge the encouragement and support given to me throughout this process by the chairperson of my committee, Dr. Rick Ingram. I cannot imagine having a better advisor and mentor for my research. Also, many thanks to all of the research assistants who helped out on this project, Kayla Meyer, Noy Phimphasone, Matthew Judah, Andrew Garcia, and Kyle Koczenowski. I would also like express gratitude to Steven Bistricky for his assistance and support, as well as Greg Siegle, who graciously offered help with several technical and logistical hurdles encountered. Lastly, I would like to thank my husband, family, and friends. You all have inspired me and, without you, I would not be where I am today. 


\section{Contents}

i

ACCEPTANCE PAGE

ACKNOWLEDGEMENTS

CONTENTS iv

ABSTRACT vi

INTRODUCTION 1

Background

Hostility-Related Cognitive Biases

Theoretical Framework

Pupillometry and Attention

Depression and Dysphoria

Current Study

METHODS

Participants

Measures

Stimulus Task

Procedure

Statistical Analyses

Data Selection, Cleaning, and Reduction Procedures

RESULTS

Observed Internal Consistency of Independent Measures

Measurement Model

Structural Model

DISCUSSION

Limitations

Strengths

Future Directions

Conclusion

REFERENCES

TABLES

Table 1: Adjectives used in the Stimulus Task

Table 2: Observed Indicator Means and Standard Deviations Table 3: Indicator Variances and Covariances 
Table 4: Loadings, Residuals, and $\mathrm{R}^{2}$ Values for Each Indicator Table 5: Correlations among Independent and Dependent Latent Constructs

Table 6: Fit Indices

Table 7: Beta Paths from the Structural Model Solution Table 8: Gamma Paths

FIGURE CAPTION

FIGURE

APPENDIX

Demographic Questionnaire

Beck Depression Inventory, Second Edition

Aggression Questionnaire

Center for Epidemiological Studies - Depression Scale

Hostile Automatic Thoughts Scale

Incidental Recall Prompt 


\begin{abstract}
A large proportion of individuals with unipolar depression experience predominantly hostile or angry as opposed to sad mood. The consideration of hostile mood states has been virtually ignored, however, in research examining cognitive processes that may contribute to the onset and maintenance of depression. To gain a better understanding of these processes, the current study explored the impact of hostility and sadness on attentional allocation patterns in dysphoria. Participants with dysphoria completed a computer task during which they rated hostile, friendly, and depressive adjectives with regard to self-relevance and other-relevance. Attentional allocation was assessed by recording reaction times associated with these ratings, measures of pupil dilation, and an incidental recall of the adjectives from the computer task. Latent constructs reflecting attention allocation to emotional and interpersonal aspects of information were regressed onto latent constructs reflecting hostility and sadness using structural equation modeling. The final model revealed that sadness, but not hostility, was a significant predictor of all attention-related constructs. Greater sadness was associated with greater attention to emotional aspects of hostile, friendly, and depressive information as well as lesser attention to self-relevant and otherrelevant aspects of information. Parameter estimates from all models suggested that pupil dilation-related markers of attention may be more closely related to reaction time than to recall. Finally, the full, untrimmed structural model revealed interesting trends regarding the role of hostility that warrant further exploration.
\end{abstract}


Effects of Sadness and Hostility on Depressive Attentional Allocation Processes

Little research on depression has considered the impact of particular mood states that often accompany this disorder such as hostility. The current study seeks to partially address this gap in the depression literature by investigating the roles of hostility and sadness on attention, one cognitive process that may have implications for the onset and maintenance of depressive disorders. To explore these processes, the current paper first reviews evidence regarding the pervasiveness of hostility in the context of depression. Then, cognitive biases that may be associated with hostility and depression are briefly reviewed along with relevant theoretical and methodological issues. Finally, hypotheses for the current study are presented and a description of the methodology, results, and implications are presented.

The mood state most often associated with unipolar depression in adults is sadness. In the Diagnostic and Statistical Manual of Mental Disorders, Fourth Edition Text Revision (DSM-IV-TR; American Psychiatric Association, 2000), "depressed mood" is the only affective symptom explicitly included in the diagnostic criteria for major depressive episode in adults (although, irritable mood is also discussed). It is somewhat common for adults meeting DSM-IV-TR criteria for a Major Depressive Episode (MDE), however, to report experiencing clinically relevant mood states other than or in addition "depressed mood," such as anger or hostility (Benazzi \& Aksikal, 2005; Fava, 1998; Pasquini, Picardi, Biondi, Gaetano, \& Morosini, 2004; Raja \& Azzoni, 2005; Scott, Ingram, \& 
Shadel, 2003). In one sample (Benazzi \& Aksikal, 2005), hostile and irritable mood was found to be a prominent affective feature in the depressive episodes of $37 \%$ of outpatients who were diagnosed with depression. Pasquini and colleagues (2004) found anger and aggression to be clinically relevant during an MDE in over $20 \%$ of a sample of outpatients with depression, whereas Fava (1998) estimated that nearly one third of outpatients with depression experience anger attacks as part of their depressive syndromes. Finally, in an impatient sample, Raja and Azzoni (2005) found hostility to be clinically relevant in 19\% of those diagnosed with unipolar depression, with no differences between male and female inpatients. Hostility is clearly an important part of the clinical presentation of depression for many individuals with depressive disorders.

Empirical evidence supports a heterogeneous view of depressive syndromes, although depression research typically "involves comparing individuals who possess a minimum number of the generally correlated symptoms with individuals who do not experience a sufficient number of these correlated symptoms" (Scott et al., 2003, pp. 233-234). There is a growing trend in depression research, however, to delineate possible subcategories of depression that account for the heterogeneous presentations observed in patients with this disorder (e.g., Koukopoulos, Albert, Sani, Koukopoulos, \& Girardi, 2005; Overall \& Hollister, 1980; Overall \& Woodward, 1975, Winokur, 1985). Overall and Hollister (1980) suggested one set of depression subtypes based on cluster analysis. They distinguished between anxious, hostile, agitated, and retarded 
depressive syndromes. Evidence of differential response to medications within these different subtypes has been found as well as epidemiological and demographic differences (Overall \& Hollister, 1980). Furthermore, these subtype classifications seem to be relevant across Western cultures, at least, with clinicians in Mexico, Italy, France, and the United States describing symptom clusters in their patients that resemble the clusters described by Overall and colleagues (Overall \& Hollister, 1980; Overall, Pull, Cabranza, \& Cassano, 1977).

Many researchers agree that hostility plays an important role in the depressive syndromes of a substantial portion of individuals diagnosed with depression. Less agreement is found in this body of literature, however, regarding how best to define "hostility." In some work, hostility has been conceptualized as a multifaceted concept, including emotional, behavioral, and cognitive pieces (e.g., Vranceanu, Gallo \& Bogart, 2006). Other researchers view hostility as largely a cognitive construct that is conceptually distinct from, but correlated with the emotional construct of anger and the behavioral construct of aggression (e.g., Smith, Glazer, Ruiz, \& Gallo, 2004; Ruiz, Smith, \& Rhodewalt, 2001). In the current study, hostility was explored in terms of its impact on depressive attentional allocation, a cognitive process likely influenced both by sadness and by hostility.

The investigations of hostility in the context of depressed mood is important not only for the sake of description, but also because of the substantial clinical and health-related implications that hostility may have for individuals 
with depression. Hostility, for example, is a well-established risk factor for the development of cardiovascular diseases (e.g., Dembroski, MacDougall, Costa, \& Grandits, 1985; Diamond, 1982; Smith, 1994; Smith et al., 2004). Furthermore, hostility may predict reduction in longevity and the increased likelihood of developing conditions such as hypertension and stroke (for a review, see Smith \& MacKenzie, 2006). Hostility may also be associated with interpersonal and occupational problems, including greater discord in close relationships (Miller, Smith, Turner, Guijarro, \& Hallet, 1996; Newton \& Keicolt-Glaser, 1995; Smith, Pope, Saunders, Allred, \& O'Keefe, 1998; Siegler et al., 2003), lower occupational status, and greater negative mood at work (Flory, Matthews, \& Owens, 1998). It is possible that this interpersonal and occupational dysfunction may at least partially explain the finding that hostility is associated with depression risk (Ingram, Trenary, Odom, Berry, \& Nelson, 2007). Finally, hostility in the context of depression may be predictive of poor prognosis (Heerlein, Richter, Gonzalez, \& Santander, 1998) and hostility in depression is a potential risk factor for increased suicidal behavior (Weissman, Fox, \& Klerman, 1973).

\section{Hostility-Related Cognitive Biases}

A great deal of published research has explored cognitive biases associated with hostility. Some of this research has focused on biases in perceptions regarding the likelihood and causes of hypothetical negative events. In one sample of college students, for example, hostile individuals perceived human-caused events as more likely than those not caused by humans, and 
perceived others to be more responsible for negative events than sad individuals (Keltner, Ellsworth, \& Edwards, 1993). In a study by Ellsworth and Smith (1988) the induction of angry feeling was associated with attributing the causes of negative events to other people, while the induction of sad feelings was not. These other-blaming tendencies are likely maladaptive, resulting in damage to social relationships and failure to engage in more adaptive coping strategies (Tennen \& Affleck, 1990). These interpersonal consequences might then exacerbate negative mood states and, for individuals with depression, contribute to the etiology or maintenance of a depressive episode.

Research exploring cognitive correlates of trait hostility suggests that hostility is associated with negative perceptions of others in a variety of social settings. For instance, greater trait hostility in one sample was associated with tendencies to interpret the actions of other individuals as intentionally aggressive and less friendly (Pope, Smith, \& Rhodewalt, 1990). Hostile individuals in another study rated a listener shown in a video of an ambiguously supportive conversation as less supportive and friendly than did their less hostile counterparts (Vranceanu et al., 2006). In a study by Epps and Kendall (2005), individuals with higher levels of trait hostility viewed a target individual as more hostile than did individuals lower in trait hostility across a variety of hypothetical situations, including benign, ambiguous, and hostile situations. Guyll and Madon (2004) found that high level of trait hostility predicted more negative appraisals of a stimulus person under an imagined situation involving self-directed threat. High 
trait hostility may also be associated with negative social information processing biases during marital conflicts (Newton \& Keicolt-Glaser, 1995). This body of research supports Barefoot's (1992) theory that possessing a "hostile other schema" may influence perceptions of others within interpersonal contexts.

Of particular relevance to the current study is research demonstrating attentional allocation patterns in individuals with hostility, depression, and dysphoria. Attentional allocation is conceptualized as a cognitive process driven by schemas (Widmayer, n.d.), and these schemas likely streamline information processing by automatically directing attention towards information that is potentially relevant and away from information that is not relevant (Neisser, 1967). The assessment of attentional allocation in psychological research is important not only because it is a marker of schemas, but also because the allocation of attention to different aspects of the environment is, itself, an important cognitive variable relevant to information processing (Ingram, Bernet, \& McLaughlin, 1994).

Guyll and Madon (2003) conducted a study exploring attentional allocation patterns associated with hostility. In their study participants were asked for a number of hostile and friendly adjectives to rate themselves, an individual they knew and liked, and a third individual they knew and disliked. Attentional allocation was assessed by rates of adjective endorsement, reaction times, and the numbers of hostile and friendly words recalled during a surprise memory task given at the end of the experimental session. Their results indicated that 
participants higher in hostility exhibited faster reaction times when rating hostile adjectives as being descriptive of others than when rating friendly adjectives as being descriptive of others. This finding suggests that quicker allocation of attention was associated with the hostile information that was most salient to these individuals. These hostile participants exhibited a lack of bias in self-description, but were slower than the control group to make decisions in self-reference trials, possibly reflecting less clarity in the self-concept of individuals with greater hostility (Guyll \& Madon, 2003). In another study exploring the impact of hostility on attention, men high in hostility exhibited greater sustained attention to negative information about others than men low in hostility, as evidenced by greater recall of negative adjectives describing a confederate following an antagonistic interpersonal interaction (Allred \& Smith, 1991). Together, the findings from these two studies suggest that hostility may be associated with the tendency to allocate attention towards negative information relevant to other people, and to exhibit sustained attention towards this information.

\section{Theoretical Framework}

Disproportionate allocation of attention to negative stimuli is a phenomenon central to many cognitive theories regarding the etiology and maintenance of depression (e.g., Beck, 1967), an idea that has gained considerable empirical support. For instance, studies show that dysphoric, currently depressed, or previously depressed individuals who have undergone a negative mood induction in the laboratory display differential allocation of attention to positive 
and negative information when compared to nondysphoric and never-depressed individuals (e.g., Beevers \& Carver, 2003; Ingram \& Ritter, 2000; Ingram \& Wisnicki, 1999; Koster, De Raedt, Goeleven, Franck, \& Crombez, 2005; Siegle, Ingram, \& Matt, 2002). Siegle, Ingram, and Matt (2002), for example, found that participants with dysphoria attended to negative words more quickly than positive words, and were slower to identify the valence (positivity versus negativity) of words than were nondysphoric participants. Findings by Koster et al. (2005) indicated that individuals exhibiting dysphoria showed greater maintenance of attention for negative words when the stimulus was presented for a period of time sufficient to allow elaborative processing. In sum, there is substantial evidence supporting attentional allocation biases in dysphoria and depression, particularly from studies in which attentional allocation processes were measured in individuals experiencing a current negative mood state (e.g., Beavers \& Carver, 2003; Ingram \& Ritter, 2000).

The cognitive biases associated with depressive affect have been studied frequently. Only a few studies, however, have explored relationships between these cognitive biases, hostility, and dysphoria. One such study by Scott and Steidtmann (2006) found that hostile cognitions were higher among dysphoric individuals who were also high in trait anger than among nondysphoric individuals and dysphoric individuals low in trait anger. Likewise, depression and hostility have been found to be associated with similar endorsements of negative self-statements (Moreno, Fuhriman, \& Selby, 1993). Scott and colleagues (2003) 
explored the role of hostility in the attributional biases of individuals with dysphoria. In their study, participants wrote essays about their own previous negative experiences. Results indicated that individuals with hostile dysphoria displayed a tendency to blame others and attribute specific causes to negative life events. In contrast, individuals with sad dysphoria made more self-blaming and global causal attributions for negative life events. Individuals with dysphoria and high hostility, furthermore, tended to blame others for these negative events even when blind raters judged the causes of these events to be more likely global and other-irrelevant (Scott et al., 2003).

The cognitive specificity perspective (see Ingram \& Hamilton, 1999; Ingram, Kendall, Smith, Donnell, \& Ronan, 1987) favors the examination of different cognitive profiles that may be associated with different affective states, such as sad versus angry or hostile mood because different qualities of affective experiences are likely related to different patterns of attention allocation. Previous work supports the idea that specific cognitive patterns may be associated with different negative affective states (e.g., Bodenhausen, Sheppard, \& Kramer, 1994; Epkins, 1996; Guyll \& Madon, 2003; Keltner et al., 1993; Scott et al., 2003). Much of the cognitive research on depression, however, continues to focus on nonspecific affective states such as anxiety or depression (Scott et al., 2003). Depression research conducted from the cognitive specificity perspective may better illuminate information processing patterns associated with the varied clinical presentations of depression. 
Research exploring the relationships between mood and attention are rooted in the spreading activation theory (Anderson, 1983). It is thought that the presence of a negative mood state will lead to a spreading of activation among interconnected stored information, thereby increasing neural network activity corresponding to other negative information associated with the negative stimuli. This idea regarding the impact of mood on neuronal activation is reminiscent of Bower's (1981) affect priming theory of mood's influence on cognition. This theory suggests that each affective state is linked to its own memory nodes and subset of cognitive processes (Bower, 1981). For instance, when someone is in a sad mood, a specific schema in this individual may be activated that is relevant to this particular mood state, increasing the accessibility to sad-relevant schemata and sad mood-related information processing. The presence of depression with hostility, thus, may be associated with the activation of different types of schemas than depression without hostility.

The cognitive specificity perspective and the affect priming theory pertain largely to the initial allocation of attention to relevant information in the environment. The depth of processing paradigm (Craik \& Tulving, 1975; Rogers Kuiper, \& Kirker, 1977), on the other hand, pertains more to the impact of information relevance on the extent to which continued information processing occurs following attentional allocation. More specifically, deeper processing of information should result when greater meaning is attached to particular pieces of information. This paradigm is supported by evidence that words are recalled more 
frequently following tasks directing attention to the semantic aspects of stimuli, compared with recall following tasks directing attention to the structural characteristics of these stimuli (e.g., word length, whether the letters composing a word are capitalized or lower case, etc.; Craik \& Tulving, 1975; Rogers et al., 1977; Ingram, Smith, \& Brehm, 1983). Some researchers posit that this differential recall takes place because attention to semantic meaning forces greater depth of information processing (Ingram, Smith, \& Brehm, 1983; Rogers et al., 1977). Information that is considered meaningful and relevant, therefore, may engender not only greater attentional allocation immediately following the presentation of information, but also more sustained attention for further information processing and memory facilitation. The current study explores the roles of hostility and sadness in both the immediate allocation of attention and also the sustained attention towards a variety of information to better understand attention processes associated with depression.

\section{Pupillometry and Attention}

Recent interest in physiological markers of cognitive processes has given rise to technologies such as pupillometry, a promising technique for assessing attention processes in depression. Pupillometry is the measurement of changes in the diameter of the eye's pupil in response to stimuli, and is a relatively inexpensive way measuring changes in brain activity (Granholm \& Steinhauer, 2004). Research suggests that both peak pupil dilation and the duration of dilation are associated with attentional allocation, memory resource mobilization, and 
cognitive load (see Beatty, 1982a for a review). Sustained pupil dilation seems to be an especially useful marker of sustained attention and information processing (Beatty, 1982b).

Measuring pupil dilation may be a useful way to assess depth of information processing in individuals with depression, dysphoria, and hostility. In their 2004 study, Siegle, Steinhauer, and Thase found that individuals with depression showed lesser pupil dilation that control participants in the seconds following trials on a non-emotional Stroop task. In another study, Siegle, Granholm, Ingram, and Matt (2001) found that, when compared to nondepressed individuals, individuals with depression showed greater pupil dilation when identifying the emotional valence of words. Moreover, pupil dilation was found to be correlated with self-reported rumination in these individuals with depression. Similarly, in a 2003 study by Siegle, Steinhauer, Carter, Ramel, and Thase, participants with depression evidenced greater sustained pupil dilation in response to an emotional task than their nondepressed counterparts. In this study, this greater sustained dilation was especially apparent in response to negative selfrelevant stimuli (Siegle et al., 2003). These outcomes suggest that individuals with depression may more deeply process emotional aspects of information relative to nondepressed individuals. Therefore, even though there may exist some disruption in attentional processes in response to non-emotional information, individuals with depression seem to attend with greater vigilance to emotional information (Siegle et al., 2004). 
Research has demonstrated the presence of attentional allocation biases in depression and dysphoria as well as hostility. Evidence from this body of research suggests, in general, that depressed mood is associated with greater attention towards negative information that is self-relevant whereas hostility is associated with greater attention towards negative information that is relevant to others. These findings that hostility and depressed mood are associated with differential attentional allocation patterns are consistent both with affect priming theory and the cognitive specificity perspective. These perspectives also support the importance of exploring attentional allocation patterns associated with hostility in the context of depressed mood. Research investigating cognitive biases associated with hostility in depression is warranted both from a theoretical perspective and also a clinical perspective since a substantial portion of individuals with depression seem to also experience clinically significant levels of anger and hostility.

\section{Depression and Dysphoria}

Studies exploring depressive phenomena commonly utilize participants that exhibit dysphoria rather than a verified depressive episode consistent with DSM-IV-TR criteria. The use of data from participants with dysphoria, a subclinical form of depressed mood, is a practice that is supported by some depression researchers and admonished by others (see Ingram \& Siegle, 2002 for a thorough discussion). A disadvantage of this practice, even if a continuous rather than categorical assumption is applied to a particular depressive phenomenon, 
includes a reduction of effect size since a continuous assumption would presume lesser effects with lesser severity of depressed mood. Many advantages are noted by Ingram and Siegle (2002), however, associated with the use of participants with dysphoria. Increased sample size is one advantage often afforded by the use of samples with dysphoria rather than depression. Furthermore, dysphoria represents a risk factor for the development of depression and this subclinical state likely represents the majority of depressed mood states experienced worldwide. In general, however, the appropriateness for any given study of sampling from individuals with dysphoria largely varies depending on the phenomena of interest (Ingram \& Siegle, 2002).

The current study utilized a sample of participants exhibiting dysphoria. This method was considered appropriate since several previous studies have demonstrated depressive attention biases using samples of individuals with dysphoria (e.g., Koster et al., 2005; Siegle, 1999; Siegle, Ingram, \& Matt, 2002). The findings from these studies suggest that depression-related attention biases represent a continuous depressive phenomenon and, thus, is should increase in effect size as a function of depressed mood severity. For continuous depressive phenomena, furthermore, research demonstrating significant effects using samples with dysphoria supports the worthiness of exploring similar phenomena in individuals meeting DSM-IV-TR criteria for depression. Sample size was an additional factor in choosing to sample individuals with dysphoria since the current study utilized statistical methods that require relatively large samples. 
Finally, attentional allocation biases associated with hostility in dysphoria could represent a particularly interesting risk factor for the development of depression since hostility may be associated with increased interpersonal discord and stress (e.g., Miller et al., 1996; Newton \& Keicolt-Glaser, 1995; Smith et al., 1998).

\section{Current Study}

The current study extended previous research by examining attentional allocation patterns associated with both sadness and hostility in the context of dysphoria. In particular, this examined hostility-related attentional allocation in depressed mood. To explore these processes, participants completed a computer task during which they indicated the relevance of a number of hostile, friendly, and depression-related adjectives to both themselves and to people they knew. For each trial, attentional allocation was assessed by measuring the RTs, peak pupil dilation, post-peak slope in pupil waveforms, and incidental recall of the task adjectives. RT, incidental recall, and pupil dilation measures were chosen to assess attentional allocation processes since these measures are unlikely to be impacted by subject reactivity effects. Data analyses, however, included measurements only from those trials in which adjectives were rated as selfrelevant or other-relevant.

I hypothesized that levels of hostility among individuals with dysphoria would impact attention to stimuli such that high levels of hostility would be associated with greater immediate and sustained attentional allocation biases towards other-relevant and hostile stimuli than lower levels of hostility. More 
specifically, I hypothesized that hostility would be more strongly associated with briefer RTs, greater maximum pupil dilation, smaller slope following peak dilation, and greater recall for these hostility-related and other-relevant stimuli than for friendly and self-relevant stimuli. Similarly, I hypothesized that more intense sadness would predict greater attention to self-relevant and depressive stimuli, as evidenced by briefer RTs, greater maximum pupil dilation, smaller slope following peak dilation, and greater recall associated with these stimuli. Previous research has provided mixed findings regarding gender differences in the expression and experience of hostility (for a review, see Fischer et al., 1993; Sharkin, 1993). I made no predictions, therefore, about gender differences in hostility and the attentional allocation processes explored in the current study. Findings indicating differential impacts of sadness and hostility in attentional allocation would be interesting in their own right, but may also support research on depression subtypes and cognitive specificity paradigms as well as suggest improvements in psychotherapeutic treatment for individuals with depression who present with predominantly hostile mood.

\section{Methods}

\section{Participants}

Data were collected from a total of 256 participants recruited from a pool of over 1,000 students enrolled in an introductory psychology course at the University of Kansas during the spring 2008, fall 2008, and spring 2009 semesters. Data associated with one participant were excluded from analyses due 
to seemingly random responses. In addition, data from 107 participants were excluded because these participants scored less than 13 on the second administration of the second edition of the Beck Depression Inventory (BDI-II; Beck, Steer, \& Brown, 1996). Responses from a total of 148 participants exhibiting dysphoria, therefore, were included in the data analyses. All students, regardless of data inclusion status, received the same credit towards class research participation requirements in exchange for their participation.

Of the 148 participants in the final sample, $61 \%$ were female $(n=90)$ and $39 \%$ were male $(n=58)$. The average age in this sample was $19.7(\mathrm{SD}=2.75)$ and participants ranged in age from 18 through 32 years. Among the participants, $78 \%(n=116)$ described themselves as Caucasian and 22\% $(n=32)$ described themselves as multiracial, African American, Latino/a, Asian, Native American, or as identifying with another racial/ethnic status. Finally, $23 \%$ of the sample $(n=$ 34) included in analyses reported using psychoactive medications.

\section{Measures}

Scores on the BDI-II were used to determine eligibility for participation in the current study and to assess severity of dysphoria in the sample. The BDI-II has yielded coefficient alphas ( 0.93 for the college students) that are higher than those for the original Beck Depression Inventory (Beck et al., 1996; Beck, Steer, \& Garbin, 1988). The BDI-II has also demonstrated good test-retest reliability, and is more closely aligned with the diagnostic criteria for depression listed in the DSM-IV-TR, thereby increasing its content validity (Beck et al., 1996). The BDI- 
II includes 21 items that address a range of depressive phenomena. Participants responded to each item by indicating on a scale of 0 through 3 the extent to which they have experienced the described phenomenon in the previous two weeks. On one item pertaining to lack of pleasure in activities, for example, $0=$ "I get as much pleasure as I ever did from the things I enjoy," 1 = "I don't enjoy things as much as I used to," 2 = "I get very little pleasure from the things I used to enjoy," and 4 = "I can't get any pleasure from the things I used to enjoy" (Beck et al., 1996). The BDI-II seems to exhibit a three factor structure, with factors described as representing negative attitude, performance difficulties, and somatic symptoms (see Osman et al., 1997). Another depression measure, therefore, was administered to assess the extent to which the participants were experiencing sadness-related depressive phenomena.

The Center for Epidemiological Studies - Depression Scale (CES-D; Radloff, 1991) was included in the questionnaire portion of the current study because it includes a subscale that reflects sadness-related depressive experiences. Radloff (1991) provided evidence for the reliability and suitability of CES-D in college samples, and another study found the sadness subscale of the CES-D (CES-D-S) to possess high internal validity (Murrell, Salsman, \& Meeks, 1995). This sadness subscale was particularly useful in the current study, therefore, since it was designed to represent a range of sadness-related depressive phenomena and has demonstrated validity as a stand-alone construct. The CES-D-S contains 7 items reflecting sadness-related emotions, thought, and behaviors. Ratings 
indicate the frequency of these phenomena during the previous week, and are selected using the following 0 to 3 Likert scale: $0=$ "rarely or none of the time," 1 $=$ "little or some of the time," $2=$ "occasionally or a moderate amount of the time," and 3 = "most or all of the time" (Radloff, 1977).

The Aggression Questionnaire (AQ; Buss \& Perry, 1992) was administered in the questionnaire portion of the study. The AQ consists of four subscales representing anger, hostility, verbal aggression, and physical aggression. This measure exhibits good construct validity, as evidenced by self-report and spousal ratings (Buss \& Perry, 1992), with high internal consistency and stability coefficients over seven months (Harris, 1997). The use of the hostility subscale in the assessment of hostile mood is supported by the confirmatory factor analysis showing support for the AQ's four-factor structure (Harris, 1995). The hostility subscale of the AQ contains eight statements (e.g., "I have become so mad that I have broken things.") that participants rated in terms of self-descriptiveness. Ratings were made based on a five-point scale with 1 = "extremely uncharacteristic of me," 2 = "somewhat uncharacteristic of me," 3 = "neither uncharacteristic nor characteristic of me," 4 = "somewhat characteristic of me," and 5 = "extremely characteristic of me" (Buss \& Perry, 1992).

The Hostile Automatic Thoughts Scale (HATS; Snyder, Crowson, Houston, Kurylo, \& Poirier, 1997) was developed to address a gap in availability of scales measuring hostile thinking style, and it was administered in addition to the AQ hostility subscale to allow further exploration of hostile cognition in 
dysphoria. The HATS includes 30 items and has exhibited high reliability (alpha = .94 , split-half reliability $=.95$ ), significant correlations with related measures, and nonsignificant correlations with measures of positive thinking (Snyder et al., 1997). On the HATS, participants rated each of the 30 items regarding the frequency with which they had the thought described, or one similar to it, in the previous week. Ratings were made based on a five-point scale with $1=$ "not at all," 2 = "sometimes," $3=$ "moderately often," $4=$ "often" and $5=$ "all the time" (Snyder et al., 1997).

\section{Stimulus Task}

To assess attentional allocation, participants completed a computer task similar to the one utilized by Guyll and Madon (2003). At the beginning of this task, participants wrote down the name of an individual they like and trust, as well as the name of an individual they dislike and distrust. They were then asked to decide, for a number of trait adjectives, whether the adjectives described themselves as well as the identified liked and disliked individuals. The participant made each decision by choosing either one of three buttons on a game controller in response to each adjective presented on the computer screen. Participants were instructed that one button indicated that the word did not describe the individual, a second indicated the word described the individual somewhat, and the third indicated the word very much described the individual. Responses were scored on a Likert scale ranging from 1 through 3 , with $1=$ does not describe the person, $2=$ describes the person a little, and $3=$ very much describes the person. The order of 
the buttons was randomized across participants, but consistent for each participant throughout their session. (See Table 1 for the lists of hostile, friendly, depressive, and nondepressive adjectives used.)

The 20 hostile and 20 friendly adjectives used in this task were those developed by Guyll and Madon for their 2003 study of attention in hostile individuals. This adjective list was balanced in terms of length and frequency of usage in the English language (Guyll \& Madon, 2003). The 20 depressive and 20 nondepressive adjectives used in the current study were those used by Ingram, Smith, and Brehm (1983). Recall of adjectives in these lists of sad and nondepressive adjectives successfully differentiated depressed from nondepressed participants in the 1983 study by Ingram and colleagues.

Each of 80 adjectives of interest were presented only once to each participant. Each of the 4 adjective groupings (20 each of friendly, hostile, sad, and neutral adjectives) were split into two lists of equal length so each adjective could be used in ratings of each person (self, liked-other, and disliked-other). Ratings of self-relevance were made by each participant using adjectives from one list associated with each of the four adjective groupings (i.e., 10 hostile, 10 friendly, and $10 \mathrm{sad}$, and 10 neutral adjectives), and ratings of other-relevance were made by each participant using adjectives of the lists not rated for selfrelevance. Ratings of the disliked other were made using the 10 adjectives from the list of hostile adjectives not rated for self-relevance as well as 5 each of the neutral and sad adjectives not rated for self-relevance. Ratings of the liked other 
were made using the 10 adjectives from the list of friendly adjectives not rated for self-relevance as well as the 5 neutral and 5 sad adjectives not rated for selfrelevance or disliked other-relevance (totaling 40 adjectives rated for selfrelevance and 20 adjectives each rated for liked other- relevance and disliked other-relevance). All adjective list assignments were determined using a random number generator. The generated numbers corresponded to 64 different E-prime experiment files, each corresponding to 1 of the 64 different permutations of adjective list assignment described above. RTs and responses for each trial were collected by E-prime. Measures of pupil dilation were collected using I-SCAN and Matlab softwares. Following the computer task, participants completed the questionnaires and a surprise incidental recall task. For the recall task, each participant was given three minutes during which they wrote any adjectives they could recall seeing during computer task.

\section{Procedure}

A double-gating procedure based on BDI-II scores was used to determine eligibility for participation in the current study. Individuals that scored at or above 13 on the BDI-II at the time of a prescreening questionnaire administration were invited to participate in the experimental session. For the second gating, data only for those participants with BDI-II scores still at or above 13 at the time of the session were included in analyses. Participants no longer scoring at least 13 on the BDI-II completed each part of the session, although their data were not included in analyses. (Each participant earned equal numbers of credits towards their class 
requirements regardless of data inclusion status.) This double-gating method of selection has been advised by some depression researchers (e.g., Ingram \& Siegle, 2002; Kendall, Hollon, Beck, Hammen, \& Ingram, 1987) to increase the likelihood that analyzed data are from participants experiencing a stable negative mood state.

Upon arriving at the session, all participants read and signed a consent form explaining the study procedures and research questions. Then, each participant completed an eye exam using a hand-held eye chart to ensure that no vision impairment was present that might interfere with completion of the computer task. Participants were permitted to wear prescription eyeglasses and contact lenses, and no participant was found to exhibit vision poorer than 20-40. After the eye exam, participants were asked to write the names of an individual they liked and trusted and an individual they disliked and trusted. Each participant was instructed to choose individuals they felt they knew well, as opposed to a celebrity or someone they'd only recently met. After choosing their liked and disliked individuals, participants completed the computer rating task during which they rated themselves, the liked other, and the disliked other in terms of 93 adjectives, 5 filler adjectives presented in a practice version of the task, 4 fillers presented just after the practice task to reduce primacy biases in recall, the 80 adjectives of interest, and 4 filler adjectives presented at the end of the task to reduce recency biases in recall. After the computer task, participants completed the packet of questionnaires and were then asked to spend three minutes listing 
adjectives they remembered viewing during the computer task. Finally, participants were debriefed and dismissed. The entire session lasted between 45 and 60 minutes for most participants.

\section{Statistical Analyses}

All research hypotheses in the current study were evaluated using structural equation modeling (SEM). I considered SEM ideal for the analysis of data from the current study since it reduces unreliability-related biases in parameter estimates, allows the simultaneous modeling of regression paths between several independent and dependent variables, and allows the full partialling of covariate variance from both independent and dependent constructs. LISREL 8.80 SEM software was used in nearly all analyses described here. The only exception entailed the use of SAS 9.2 statistical software for the multiple imputation of missing data and open source spreadsheet software, Open Office Calc 3.1, for the calculation of observed indicator means, observed indicator standard deviations, and sample demographic statistics.

\section{Data Selection, Cleaning, and Reduction Procedures}

Several steps associated with data cleaning were required before analyses

for the current study could be conducted. E-prime was used to collect RT data and trial responses and Matlab was used to collect pupil dilation data. First, all pupil dilation graphs were inspected in Matlab to ensure reliable collection of pupil data. Pupil data from three participants were excluded from further analyses due to erratic pupil data and data from five participants were excluded due to 
incomplete pupil data. Next, using spreadsheet software, peak dilation and extent of downward slope from 3 seconds through 12 seconds following trial onset were computed. These two characteristics of the pupil wave form are particularly useful in measuring attention allocation (Siegle, 1999). These per trial peak dilation and slope variables were then matched to their respective E-prime response and RT data.

Indicators used in the structural equation model were derived using only trials for which participants indicated that the trial word was descriptive of the relevant individual. This was to ensure that only self and other-relevant data were modeled, those for which biases in attention would be expected. For each individual, average RTs, response, dilation peaks, and pupil waveform slopes for these self-and other-relevant trials were then computed for each of the following seven categories: self-relevant hostile information trials, self-relevant friendly information trials, self-relevant depressive information trial, liked other-relevant friendly information trials, liked other-relevant depressive information trials, disliked other-relevant hostile information trials, and disliked other-relevant depressive information trials. These averages were then matched by participant with the questionnaire data to create a single dataset containing all variables. Pupil waveform slopes from 3 through 12 seconds following trial onset typically exhibit a negative trajectory. Raw slope calculations were multiplied by -1.0 , however, to generate positive values for ease of imputation. A higher loading from a slope indicator onto a latent attention-related construct, therefore, is 
expected to be positive, with larger values indicating greater negative slope in the waveform. Also, the averages for RT were computed in seconds instead of milliseconds. Peak dilation and waveform slope values were similarly rescaled. Peak dilation values were multiplied by 10 and the slope values by 100 . All variable rescaling was performed to increase stability of parameter estimates (particularly factor loadings) and to aid in imputation since values associated with raw pupil data are very small and RT values, measured in milliseconds, are quite large.

Three parcels each for the Hostility and Sadness independent latent constructs were derived using the item-to-construct balance approached described in Little, Cunningham, Shahar, and Widaman (2002). This approach entailed the assignment of HATS and CES-D-S items to three parcels for each construct such that one each of the three indicators, those with the highest loadings on their relative latent constructs, are assigned to each parcel in a particular order. Then, one each of the three indicators with the next highest loadings are assigned to each parcel in an inverse order, and the rest of the items are similarly balanced across parcels. Items associated with each parcel were then summed to attain the parcel scores for each individual. The goal of this approach is to create, for each latent construct, three parcels with reasonably equivalent loadings (Little et al., 2002). Parcels were also created from the AQ-hostility subscale and the AQ-anger subscale. The constructs built from these parcels, however, failed to model well in a measurement model including all potential model constructs (the loadings were 
quite tiny and, therefore, the constructs exhibited unreasonable small covariances with conceptually related constructs). These constructs were dropped from analyses and the construct built from the HATS parcels was chosen instead as a primary independent variable.

The dataset for the current study contained missing data due primarily to equipment errors and researcher error in equipment operation. A minority of missing data were due to participant omissions on questionnaires and, for a few participants, a lack of responses indicating relevance of stimuli in particular categories. A few participants, for example, indicated that none of the depressive adjectives presented in the liked other-relevant portion of the computer task were descriptive of the person they chose as their liked other. Most of the missing data were due to researcher and equipment errors and, therefore, could be considered missing at random. (See Allison, 2002 for a discussion of different types of missingness in data.) For missingness due to lack of relevance of a set of stimuli, response averages of 1 (indicating all adjectives in a particular category were not relevant) were maintained to improve reliability of the imputed RT and pupil data associated with these trials. After attaining a single dataset containing all variables, the PROC MI procedure in SAS 9.2 was used to create five imputations. This number of imputations was chosen based on Rubin's 1987 guidelines. I used the MCMC option in SAS to allow for control of certain aspects of the imputation procedure. The MCMC allowed specifications of maximum and minimum imputation values for the RT variables and pupil variables. Also, I 
specified 500 burn-in iterations be completed prior to the first imputation and 200 iterations between each imputation using the "nbiter" and "niter" options in the SAS PROC MI. The procedure used was similar to an example given by Allison (2002, p. 43). SAS output following the PROC MI procedure indicated approximately $15 \%$ missingness in the original dataset.

Before constructing the measurement model, I examined descriptive data related to each individual indicator (see Table 2 for observed means and standard deviations) and examined the distribution of responses for each indicator. This data screening revealed no severe nonnormality in the indicators. Univariate normality is required in SEM if maximum likelihood (ML) estimation is used because multivariate normality is an assumption inherent in ML estimation procedures. If univariate normality is violated, the assumption of multivariate normality is also violated (Brown, 2005). The assumption of multivariate normality for these data was further supported by LISREL-generated QQ plots.

All models for the current study included ten latent constructs representing two independent constructs, Hostility and Sadness, as well as six dependent constructs, hostile information (Host), friendly information (Friendly), depressive information (Sad), self-relevant information (Self) liked other-relevant information (Liked), and disliked other-relevant information (Disliked). The Hostility and Sadness constructs had three indicator parcels each. Each of the six dependent constructs had either 8 or 12 indicators each. The Self construct was represented by 12 indicators, 4 indicator types each (RT, memory, peak pupil 
dilation, and dilation slope) from the self-relevant hostile information, selfrelevant friendly information, and self-relevant depressive information trial averages. The Liked construct had eight indicators and was represented by these same four indicator types from the liked other-relevant friendly information and liked other-relevant depressive information. The Disliked construct was similarly constructed with four indicators each representing attention to disliked otherrelevant hostile information and disliked other-relevant depressive information. The Sad construct was represented by these same four indicator types from the self-relevant depressive, liked other-relevant depressive, and disliked otherrelevant. This is the second and final construct represented by 12 indicators. The Friendly construct is an example of a dependent construct with only eight indicators, four representing attention to self-relevant friendly information and four representing liked other-relevant friendly information. Finally, the Host construct was similarly constructed with four indicators of self-relevant hostile information and four of disliked other-relevant hostile information. To build constructs in this way, each indicator loaded both onto its relevant person-related latent construct (Self, Liked, or Disliked) as well as its relevant emotional valence-related latent construct (Host, Friendly, or Sad).

The factor identification method was used for model identification and scale setting in both the measurement and structural models. The factor identification method entails setting the scale of latent constructs by fixing their variances at 1.0. In addition to setting the scale, factor identification produces 
standardized estimates of covariances (i.e., correlations) among latent constructs (Brown, 2005) by allowing each HATS and CES-D-S parcel to load onto its respective independent latent construct. Each dependent indicator loaded onto both its respective person-related and its respective valence-related dependent constructs. Also estimated in this model were the unique variances associated with each indicator and the correlations among the latent constructs. The variances of the latent constructs, as previously mentioned, were fixed at 1.0 for scale-setting and identification purposes.

\section{Results}

Parameters in each model were estimated by LISREL 8.80 software based on the variance/covariance matrix derived from the use of the raw data files. (See Table 3 for the full observed variance/covariance matrix.) Parameter estimates were combined across the five imputations using a parameter estimate pooling program available online (Geldhof, n.d.).

\section{Observed Internal Consistency of Independent Measures}

In the sample for the current study, item responses on the HATS exhibited good internal consistency (Chronbach's alpha $=0.95)$, as did item responses on the CES-D-S (alpha $=0.77)$. These internal consistency estimates, furthermore, changed very little when the consistencies of parceled responses were estimated (alpha $=0.94$ for the HATS parcels; alpha $=0.80$ for the CES-D-S parcels). BDIII responses among the current sample with dysphoria exhibited lower internal consistency $($ alpha $=0.74)$ than did responses in the general college student 
sample collected by Beck and colleagues $(1996$; alpha $=0.93)$. The lower alpha for responses made by those in the current sample, however, was expected due to a restriction of range in BDI-II responses. This restriction in range is an inevitable result of selecting for participation in the study only those individuals exhibiting elevated BDI-II total scores.

Item responses associated with the anger subscale of the AQ (AQA) exhibited good internal consistency (alpha $=0.82)$. The Chronbach's alpha associated with the hostility subscale of the $\mathrm{AQ}(\mathrm{AQH})$, however, was lower than expected $(\mathrm{alpha}=0.71)$. The internal consistency estimates associated with the parceled AQ subscale indicators did not exhibit improvement in internal consistency, furthermore, over those associated with the non-parceled item responses (for AQA parcels, alpha $=0.75$; for $\mathrm{AQH}$ parcels, alpha $=0.69$ ).

\section{Measurement Model}

A measurement model was constructed using LISREL software by allowing each indicator to load onto its respective latent construct(s). Values reflecting the strengths of each loading were estimated in the measurement model. Also estimated in this model were the unique variances of the indicators and the correlations among the latent constructs. The sample for the current study was relatively small $(\mathrm{n}=148)$. The CFI and RMSEA fit indices, therefore, were the most carefully considered indices of model fit since these fit indices tend to be less biased by small sample size than are other popular indices such as NNFI and $\chi^{2}$ (Fan, Thompson, and Wang, 1999). By convention, the cutoff RMSEA for 
acceptable fit is .08 (smaller values indicate better fit) and the cutoff CFI is 0.9 (greater values indicate better fit; e.g., Kline, 2005). The measurement model for the current study demonstrated acceptable fit as indicated by both the RMSEA and CFI $\left(\chi^{2}(471, n=148)=878.21, p=<.001\right.$, RMSEA $=.067,90 \% \mathrm{CI}$ for $\mathrm{RMSEA}=.058-.074, \mathrm{NNFI}=0.91, \mathrm{CFI}=0.92)$. Inspection of the indicator loadings (see Table 4) confirmed that most indicators loaded as expected on their respective latent constructs and that most were statistically significant. The loadings associated with the HATS and CES-D-S parcels, reaction time (RT), max pupil dilation (Max), and slope of dilation waveforms (Slope) were particularly consistent with expectations. Loadings for the stimulus recall indicators exhibited less consistency, both in terms of expected loadings and also valence of loadings. Several loadings associated with the recall indicators, furthermore, failed to demonstrate statistical significance. No modification index exceeded $10 \%$ of the $\chi^{2}$ value, suggesting that no changes to the measurement model were necessary. (See Table 5 for estimates of latent construct covariances and Table 6 for fit indices.)

\section{Structural Model}

Beta paths representing the dependent constructs regressed onto the independent constructs were freed and the parallel latent covariance estimates constrained to convert the measurement model into a structural model. Two covariates, BDI-II scores and gender (as indicated by questionnaire response), were also included. I freed directional paths (gamma paths) from these covariate 
constructs to both the independent and dependent latent constructs, allowing the partialling of covariate effects from both the independent latent constructs. Very little change in model fit was observed between the measurement model and the structural model. The structural model continued to exhibit acceptable fit $\left(\chi^{2}(523\right.$, $n=148)=965.30, p=<.001, \mathrm{RMSEA}=.066,90 \% \mathrm{CI}$ for $\mathrm{RMSEA}=.058-.075$, $\mathrm{NNFI}=0.90, \mathrm{CFI}=0.92$ ). Many of the beta paths exhibited statistical significance (see Table 6 for fit indices and Table 7 for beta paths). More specifically, each of the beta paths associated with the Sadness construct exhibited statistical significance whereas none of the beta paths associated with the Hostility construct exhibited significance. Each gamma path exhibited statistical significance. (See Table 8 for Gamma paths and Figure 1 for an illustration of the structural model.)

Three out of the six statistically significant beta paths suggested that higher sadness was associated with greater attentional allocation towards emotionally-relevant aspects of information. The other three significant beta paths suggested that higher sadness was associated with less attentional allocation towards self-relevant and other-relevant aspects of information. It should also be noted that the beta paths associated with the effect of hostility on the dependent constructs, when considered alongside the statistically significant beta paths associated with the effect of sadness, exhibited interesting patterns (see Table 7). These patterns indicated a tendency for the Hostility construct to be associated with greater attention to self-relevant and other-relevant information and less 
attention to the emotional valence of information, a general pattern opposite to that of the beta paths from the Sadness construct. These hostility-related beta paths, however, did not exhibit significance at the $\mathrm{p}<.05$ level.

Each of the 16 gamma paths in the structural model exhibited statistically significance. These gamma paths suggested that higher BDI-II scores were associated with higher hostility and sadness, greater attentional allocation towards self-relevant and other relevant information, and less attentional allocation towards emotionally-relevant information. Additionally, males tended to exhibit greater hostility and greater attention to emotional-relevant aspects of information, whereas women tended to exhibit greater sadness and greater attention to self-relevant and other-relevant aspects of information.

\section{Discussion}

Parameter estimates derived from the structural equation model in the current study did not support many of my hypotheses. These estimates did reveal significant findings, however, regarding sadness as a predictor of attentional allocation patterns in a sample of college students exhibiting dysphoria. Hostilityrelated patterns examined in the model were also of interest, particularly when considered alongside the sadness-related patterns, but they were not statistically significant and not as expected. The final structural model exhibited significant tendencies for sadness to predict increased attention to emotional aspects of depressive, hostile, and friendly information, and decreased attention to selfrelevant and other-relevant aspects of information. The patterns associated with 
hostility revealed by this model reflected opposite tendencies, but these hostilityrelated associations did not exhibit statistically significance. The patterns revealed regarding the impact of both hostility and sadness on depressive attention processes, however, are certainly worth further attention.

The structural model reported here suggested particular patterns associated with the impact of sadness and hostility on depressive attention. These patterns suggested that greater sadness was associated with greater attention to emotional aspects and less attention to interpersonal aspects of information, whereas greater hostility tended to be associated with greater attention to interpersonal aspects of information and less attention to emotional aspects of information. The effects of sadness and hostility, therefore, may be opposite to one another in terms of their impact on attention to emotional and interpersonal aspects of information. Regression paths related to hostility were not statistically significant, however, so these statements are made with great caution. The pattern is interesting enough to at least tentatively offer support to the cognitive specificity paradigm. The hostility-related patterns exhibited by the structural model were completely opposite to the sadness-related patterns, reflecting differential effects of sadness and hostility on attention to different emotional and interpersonal aspects of information. Additional exploration of this pattern is required, however, before stronger statements of support can be offered.

Another interesting finding that emerged was the tendency for higher sadness to be associated with increased attention not only to depressive aspects of 
information, but to all relevant emotional aspects of information. This finding suggests that, when variance associated with interpersonal aspects of information is removed, sadness is associated with attention to both positive and negative emotional information. Siegle (1999) reported a similar finding in his study of attention to self-relevant stimuli in dysphoria. In his paper, Siegle stressed the importance of studying attention to information identified as relevant to participants with depressed mood, and suggested that this increased attention to both positive and negative information may result from a depressive tendency to associate a range of incoming emotional information with negative self-relevant information. In support of this hypothesis, he found that participants with dysphoria less frequently identified positive words as positive in valence, compared with control participants, and they exhibited greater attention to both relevant positive and relevant negative information (Siegle, 1999). The current study, similarly to Siegle's (1999), revealed that sadness in the context of dysphoria was associated with greater attentional allocation not only towards relevant depressive information, but also towards relevant hostile and friendly information.

The finding that higher sadness was associated with less attentional allocation to self-relevant aspects of information is difficult to explain, but interesting possibilities are worth consideration and further study. For all attention measurements in the current study, the variance associated with emotional valence and the variance associated with self-relevance were allowed to load onto separate 
latent constructs. No latent constructs, however, represented combinations of person and emotional valence. A combined factor representing attention to selfrelevant negative information, for instance, may be required to demonstrate an effect of sadness on self-relevant information in depression and dysphoria. With sufficient numbers of participants, a model could be constructed using similarly combined constructs such that the dependent factors represent attention to the following: self-relevant sad information, self-relevant hostile information, selfrelevant friendly information, disliked other-relevant sad information, disliked other-relevant hostile information, liked other-relevant sad information, and liked other-relevant friendly information.

If individuals with depression and dysphoria exhibit the tendency to associate many kinds of information with negative material, as suggested by Siegle (1999), it is possible that studies demonstrating depressive self-focused biases have inadvertently confounded measures of self-relevant bias with the extent to which a range of emotional information triggers negative schemas in depressed states. These biases, in other words, may be attributable to attention to the emotional content of the information rather than to the self-relevance of the information. Another possibility is that the effect of depressed mood on attention to negative self-relevant information in previous studies was confounded with levels of participant hostility. In these samples, the depressed individuals were likely higher in hostility than their nondepressed counterparts and it could be this higher hostility that was truly associated with the increased attention to self- 
relevant negative information. In the current sample, for instance, greater severity of dysphoria was associated with greater hostility. Each of these possibilities should be further explored before conclusions regarding these possibilities are drawn.

The hostility-related patterns exhibited by the structural model leave open the possibility that hostility has an important role to play in depressive cognitive processes. For instance, the grouping of all participants into a single group with both genders may have obscured the extent to which gender impacts the ability of hostility to predict attentional allocation patterns. A two group model with males in one group and females in the other, for instance, may reveal hostility-related effects that are trivial in one gender, but statistically significant in the other. This two-group model would allow more a thorough examination of gender differences in the relationships between sadness, hostility, and attentional allocation. Hostility in this two-group model may emerge as a significant predictor of attention in one or both genders. Unfortunately, the sample size in the current study did not allow for two-group modeling.

Gender was included as a covariate in the analyses here rather than as a grouping variable. This did not allow an ideal exploration of gender differences in the ways in which sadness and hostility predict attention in dysphoria. The findings here, however, did corroborate previous findings of gender differences in hostility such that men exhibited more hostile thinking than did women.

Findings from the current study highlight the importance of including 
scores from measures of depression severity as covariates when examining relationships among depressive phenomena in the context of depressed mood. Any given depressed state can include many components including sadness, anhedonia, anxiety, hostility, hopelessness, and countless others. Researchers interested in these particular depressive phenomena in the context of depression, rather than the entirety of the depressed state, per se, should include scores from a depression scale as a covariate to ensure that the effects of the particular depressive phenomena of interest are not confounded with other, overlapping features. In the current study, for example, it was important to include depression severity as a covariate to ensure that the revealed effects of sadness and hostility were not actually caused by overlapping phenomena such as guilt, the sense of worthlessness, or suicidal ideation. Studies with larger samples should also consider including measures of anxiety and trait anger as covariates since these phenomena have exhibited significant overlap with depression (Kendall \& Watson, 1989; Scott \& Steidtmann, 2006).

Of interest, methodologically, is the finding that pupil dilation indices and reaction time consistently loaded as expected onto the dependent attention-related latent constructs, but recall did not. Recall indicators exhibited less consistency in loading; exhibiting some positive loadings, some negative loadings, and several nonsignificant loadings on the dependent latent constructs. The reason for this finding is unclear.

The depth of processing paradigm suggests emotionally salient 
information should be associated with both greater proximal attentional allocation and also greater distal information processing. The distal processing should thus produce greater recall for emotional information judged as self-relevant or otherrelevant. This prediction was not supported, however, by results from the current study.

Future research should explore the role of the time lapsed between encoding and recall on information recalled by individuals with depression and dysphoria. In the current study, approximately 20 minutes typically elapsed between the time participants finished the computer rating task and the time they began the incidental recall task. It is possible that recall variables would exhibit loadings onto latent attentional constructs more similarly to those from pupillary and reaction time measures when the time between information encoding and recall is reduced. Another possibility is that recall after a longer lapse would be facilitated by induced sympathetic nervous system activation following information encoding. Bradley, Miccoli, Escrig, and Lang (2008) found that pupil dilation in response to emotional information was correlated with sympathetic activation, as measured by skin conductance. Smeets, Otgaar, Candel, and Wolf (2008) found that recall of emotional information after a longer duration of time, though, is enhanced when sympathetic nervous system arousal is induced just following the presentation of this information rather than just before. Including a stressor to induce increased sympathetic activation following the encoding of relevant emotional information, therefore, may engender greater subsequent recall 
for this information when the time lapse between encoding and recall is of concern.

Differences in implicit versus explicit recall of information would also be interesting to explore in studies using recall as a marker of attentional allocation to emotional and interpersonal aspects of information. Implicit recall is measured by asking participants to complete tasks in which previously presented information is reproduced without explicitly asking them to recall the information. Examples of commonly used implicit recall tasks include word stem completions and free associations (see Watkins, Martin, \& Stern, 2000). Transfer appropriate processing theory suggests that implicit word recall following a conceptually driven encoding task (in which participants attend to word meaning such as choosing word valence or rating word relevance) should be greatest when using an implicit memory task that is also conceptually driven (e.g., free association). Following a computer task in which words are rated for selfrelevance and/or other relevance, therefore, implicit recall may be best demonstrated by conceptually driven implicit recall tasks (Watkins et al., 2000). Unknown, however, is the extent to which implicit recall indicators may load similarly onto attentional latent constructs, compared with reaction time and pupil dilation indicators.

\section{Limitations}

The largest limitation in the current study was its sample size. The required sample size for SEM increases as the complexity of the model increases 
because reliable estimates require at least as many participants as freely estimated parameters (Brown, 2005). Small sample size could be related to some of the nonsignificant hostility-related patterns in the current study. Another possibility is that there is no effect of hostility on these attentional processes, but studies with larger samples should be conducted before firm conclusions can be drawn.

The imputation of attentional data corresponding to blocks for which participants indicated no adjectives were relevant to the rated person represents another significant limitation in data analyses for the current study. Imputation was performed so that maximum likelihood (ML) estimation, the most common estimation method used in structural equation modeling, could be utilized. I considered ML estimation important for the analyses of data from the relatively small current sample, furthermore, to enable the estimation of well known fit indices appropriate for small samples in structural equation modeling (i.e., the CFI and RMSEA indices). Structural equation modeling can be conducted with missing data present if full information maximum likelihood (FIML) estimation is utilized. Aside from chi square, however, no fit statistics reported here are provided by LISREL when using the FIML estimation method. The imputation of data in place of blocks rated irrelevant, however, may have inadvertently introduced biases in the estimation of parameters. The amount of data missing due to irrelevance of stimuli, however, was small and, thus, I suspect that any biases were minimal. Furthermore, the estimates provided in the current study are likely less biased than those that would be attained using statistical techniques, such as 
traditional regression analyses, that do not correct for unreliability of measurement, allow the full partialling of covariate effects, or allow the modeling of all predictive relationships simultaneously.

A number of improvements in model specification with similar data could be achieved with larger sample sizes. First, it may be interesting to specify the dependent latent constructs such that each construct represents a particular emotion-person combination. A second benefit of a larger sample size would be the ability to include independent latent constructs representing, for example, the interaction between sadness and hostility. Third, multi-trait multi-method (MTMM) models are ideal for estimating two or more latent constructs using multiple methods. MTMM models include additional latent constructs associated with each method of measurement. Using the current study as an example, latent constructs could be formed representing reaction time, recall, peak pupil dilation, and post-peak pupil waveform slope. Over 500 participants would have been required, however, for modeling from the current study to be modified such that a third, anxiety-related covariate were included, a hostility X sadness interaction construct were included, MTMM modeling techniques were used, and separate groups representing males and females were compared.

The structural equation models described here demonstrated adequate fit, but better fit $($ RMSEA $<.05$, NNFI and CFI $>.95)$ would be ideal. The inability to construct a two-group and MTMM model likely impacted model fit adversely. Future studies employing larger sample sizes and modeling of greater complexity 
should explore this possibility.

Another modeling-related limitation of the current study was the inability to use latent factors associated with the anger and hostility subscales of the AQ as predictors of attention processes in dysphoria. Parcels associated with the hostility and anger subscales of the AQ (three parcels associated with each subscale) were constructed using the same item-to-construct balancing approach as was used in the parceling of CES-D-S and HATS scores. These AQ subscales, furthermore, have demonstrated good factor structure in previous research (Harris, 1995). Modeling difficulties were consistently encountered in analyses for the current study, however, when attempting to include latent factors representing AQ subscales using the parceled indicators. It is likely that the lower internal consistencies exhibited here by the anger and hostility AQ subscales contributed to these modeling difficulties.

\section{Strengths}

A primary strength of the current study was the use of structural equation modeling for analyses, even if the sample size did not allow for ideal model specification. This technique made possible the modeling of a large number of relationships between latent constructs simultaneously without resorting to the estimate-biasing practice of mean splitting (see MacCallum, Zhang, Preacher, \& Rucker, 2002). Also, including all regression paths in a single model reduces the dangers of inflating Type 1 error rates. Type 1 error reflects rejecting a null hypotheses that are actually true (see Gravetter \& Wallnau, 2007), and the 
reduction Type 1 error risk represents a major strength of SEM (Brown, 2005).

The modeling of latent constructs rather than observed variables, furthermore, reduces biases in estimation due to the unreliability of psychological measurements. Finally, in the current study, parameter estimates generated using SEM raised interesting questions about the use of recall as a marker of attentional allocation.

The use only of data associated with self-relevant and other-relevant information represents an additional strength of the current study. Some have suggested that the common practice of using normed stimuli that may or may not be relevant to participant schemas may weaken the results of studies that do not assess their relevance since these stimuli may be too general to activate individualistic depressive schemas among participants (e.g., Siegle, 1999). The exclusion of trials associated with non-relevant information likely allows inferences about depressive cognitive processes from the current study that are more germane to those processes contributing to the etiology and maintenance of depression and dysphoria.

A third notable strength of the current study is the use of several markers, including reaction time, pupil dilation, and recall, to assess attentional allocation. Each of these markers has been used often in research on attentional allocation processes, but researchers do not commonly use all of these markers in a single study. The methodological representativeness of the current study increases the likelihood that the results are reliable. 


\section{Future Directions}

Many lines of research inquiry could contribute substantially to knowledge about the role of different mood states in the context of depression and dysphoria. The current study explored the role of hostility and sadness in depressive attentional allocation processes, but more research is needed to further explore the role of hostility, gender, anxiety, and other relevant phenomena in these processes. A study with a larger sample, for instance, could include several predictors of attentional allocation, explore relevant interactions, and could explore potentially important group differences in the ways in which different depressive phenomena impact the allocation of attention. Research on the impact of hostility on other depressive cognitive processes, such as rumination, memory accessibility, and the processing of threat-related cues may also yield important findings.

A great deal of research, for good reason, has explored risk factors associated with depression. Very little is known, however, about factors that predict the development of hostility in depression. The lack of research on this topic represents a large gap in our understanding of the development of mood disorders. Several factors are potentially important in differentiating etiological processes in depression with and without significant hostility, including gender as well as differential biological factors such as genetic polymorphisms or neurotransmitter functioning, parent-child interactions, or life events. Clearly we have a long road ahead in our exploration of hostility in the context of depressed 
states, a phenomenon relevant to the experiences of many individuals with depressive disorders.

One way hostility may contribute to the etiology and maintenance of depression in some individuals is through the increase of interpersonal discord. For instance, interpersonal cues may be more likely to be attended to and interpreted as insidious by individuals with depression and hostility, as compared to individuals with depression but without hostility. It is also possible that these individuals higher in hostility would be more likely to end relationships or confront others following negative interpretations of benign cues. These hostilityrelated interpersonal styles, if present, may be associated with intense social isolation when depressed and, therefore, longer depressive episodes, increased rumination, and decreased social support. The only research currently available on hostility-related interpersonal styles explores these styles outside the context of mood disorders. Therefore, evidence is not currently available to support these speculations regarding the impact of hostility in the context of depression. Hostility is largely an interpersonal construct, however, and interpersonal styles associated with hostility in depression represent an important area for future research efforts.

\section{Conclusion}

The current study explored the relationships between sadness, hostility, and attention processes in the context of dysphoric mood. Hypotheses regarding these relationships were largely unsupported, but the models revealed several 
interesting patterns. First, sadness significantly predicted all attention allocation constructs. Greater sadness was associated with greater attention to emotional aspects of hostile, friendly, and depressive information. Greater sadness was also associated with less attention to self-relevant and other-relevant aspects of information. Additionally, peak pupil dilation and slopes in dilation waveforms were more closely related to reaction time as measures of attentional allocation than to explicit recall markers. Finally, interesting patterns regarding the role of hostility in these attention processes were revealed such that hostility tended to be associated with less attention to emotional aspects of information, but greater attention to interpersonal aspects of information. Future studies should explore similar models, particularly those that including interactions between sadness and hostility, and those that more thoroughly addressing gender differences. The role of hostility in a range of depressive cognitive and interpersonal phenomena should also be explored, as should differential factors that may lead to the development of depressed states with and without clinically relevant levels of anger and hostility. The exploration of these hostility-related phenomena in depression would be a welcome step towards addressing the complex nuances in the clinical presentation of this pervasive, burdensome disorder. 


\section{References}

Allison, P.D. (2002) Missing Data. Thousand Oaks, CA: Sage.

Allred, K. D., \& Smith, T. W. (1991). Social cognition in cynical hostility.

Cognitive Therapy and Research, 15, 399-412.

American Psychiatric Association. (2000). Diagnostic and statistical manual of mental disorders. ( $4^{\text {th }}$ ed., text revision). Washington, DC: Author.

Anderson, J. R. (1983). A spreading activation theory of memory. Journal of Verbal Learning and Verbal Behavior, 22, 261-295.

Barefoot, J. C. (1992). Developments in the measurement of hostility. In H. S. Friedman (Ed.), Hostility, coping, and health (pp. 13-31).

Washington, DC: American Psychological Association.

Beatty, J. (1982a). Task-evoked pupillary responses, processing load, and the structure of processing resources. Psychological Bulletin, 91(2), 276292.

Beatty, J. (1982b). Phasic not tonic pupillary responses vary with auditory vigilance performance. Psychophysiology, 19(2), 167-172.

Beck, A. T. (1967). Depression: Clinical, experimental, and theoretical aspects. New York: Harper \& Row.

Beck, A. T., Steer, R. A., \& Brown, G. K. (1996). Manual for the Beck Depression Inventory, 2nd ed. San Antonio, TX: The Psychological Corporation.

Beck, A. T., Steer, R. A., \& Garbin, M. G. (1988). Psychometric properties of the 
Beck Depression Inventory: Twenty-five years of evaluation. Clinical Psychology Review, 8, 77-100.

Beevers, C. G., \& Carver, C. S. (2003). Attentional biases and mood persistence as prospective predictors of dysphoria. Cognitive Therapy and Research, 27, 619-637.

Benazzi, F. (2003). Major depressive disorder with anger: A bipolar spectrum disorder? Psychotherapy and Psychosomatics, 72, 300-306.

Benazzi, F., \& Akiskal, H. (2005). Irritable-hostile depression: Further validation as a depressive mixed state. Journal of Affective Disorders, 84(2-3), 197-207.

Bodenhausen, G. V., Sheppard, L. A., \& Kramer, G. P. (1994). Negative affect and social judgment: The differential impact of anger and sadness. European Journal of Social Psychology, 24, 45-62.

Bower, G. (1981). Mood and memory. American Psychologist, 36, 129-148.

Bradley, M. M., Miccoli, L., Escrig, M. A., \& Lang, P. J. (2008). The pupil as a measure of emotional arousal and autonomic activation. Psychophysiology, 45, 602-607.

Brown, T. A. (2005). Confirmatory factor analysis for applied research (4 $4^{\text {th }}$ ed.). New York: Guilford Press.

Buss, A. H., \& Perry, M. (1992). The Aggression Questionnaire. Journal of Personality and Social Psychology, 63, 452-459.

Craik, F., \& Tulving, E. (1975). Depth of processing and the retention of works in 
episodic memory. Journal of Experimental Psychology: General, 104, 268-294.

Dembroski, T. M., MacDougall, J. M., Costa, P. T., \& Grandits, G.A. (1989).

Components of hostility as predictors of sudden death and myocardial infarction in the Multiple Risk Factor Intervention Trial. Psychosomatic Medicine, 51, 514-522.

Diamond, E. L. (1982). The role of anger and hostility in essential hypertension and coronary heart disease. Psychological Bulletin, 97, 410-433.

Ellsworth, P. C., \& Smith, C. A. (1988). From appraisal to emotion: Differences among unpleasant feelings. Motivation and Emotion, 12, 271-302.

Epkins, C. C. (1996). Cognitive specificity and affective confounding in social anxiety and dysphoria in children. Journal of Psychopathology and Behavioral Assessment, 18, 83-101.

Epps, J., \& Kendall, P. C. (1995). Hostile attribution bias in adults. Cognitive Therapy and Research, 19, 159-178.

Fan, X., Thompson, B., \& Wang, L. (1999). The effects of sample size, estimation methods, and model specification on SEM fit indices. Structural Equation Modeling: A Multidisciplinary Journal, 6, 56-83.

Fava, M. (1998). Depression with anger attacks. Journal of Clinical Psychiatry, 59[suppl 18], 18-22.

Fischer, P. C., Smith, R. J., Leonard, E., Fuqua, D. R., Campbell, J. L., \& Masters, M. A. (1993). Sex differences on affective dimensions: Continuing 
examination. Journal of Counseling and Development, 71, 440-443.

Flory, D. J., Matthews, K. A., \& Owens, J. L. (1998). A social information processing approach to dispositional hostility: Relationships with negative mood and blood pressure elevations at work. Journal of Social and Clinical Psychology, 17, 491-504.

Geldhof, G. J. (n.d.). Rubin's Rules Pooler for LISREL Estimates. Retrieved November 1, 2009 from http://www.quant.ku.edu/openfiles/rubins_rules.SAS.

Granholm, E., \& Steinhauer, S. R. (2004). Pupillometric measures of cognitive rand emotional processes. International Journal of Psychophysiology, $52,1-6$.

Gravetter, F. J., \& Wallnau, L. B. (2007). Statistics for the Behavioral Sciences ( $7^{\text {th }}$ ed.). Bellmont, CA:Thompson Wadsworth.

Guyll, M., \& Madon, S. (2003). Trait hostility: The breadth and specificity of schema effects. Personality and Individual Differences, 34, 681-693.

Guyll, M., \& Madon, S. (2004). Effects of trait hostility and self-relevance of social information processing. Personality and Individual Differences, $26,263-276$.

Heerlein, A., Richter, P., Gonzalez, M., Santander, J. (1998). Personality patterns and outcomes in depressed and bipolar disorders. Psychopathology, $31,15-22$.

Ingram, R. E., Bernet, C. Z., \& McLaughlin, S. C. (1994). Attentional allocation 
processes in individuals at risk for depression. Cognitive Therapy and Research, 18, 317-332.

Ingram, R. E., \& Hamilton, N. (1999). Evaluating precision in the social psychological assessment of depression: Methodological considerations, issues, and recommendations. Journal of Social and Clinical Psychology, 18, 160-180.

Ingram, R. E., Kendall, P. C., Smith, T. W., Donnell, C., Ronan, K. (1987). Cognitive specificity in emotional distress. Journal of Personality and Social Psychology, 53, 734-742.

Ingram, R. E., \& Siegle, G. J. (2002). Contemporary methodological issues in the study of depression: Not your father's Oldsmobile. In I. H. Gotlib \& C. L. Hammen (Eds.), Handbook of Depression (2nd ed., pp. 86-114). New York: Guilford Press.

Ingram, R. E., Smith, T. W., \& Brehm, S. S. (1983). Depression and information processing: Self-schemata and the encoding of self-referent information. Journal of Personality and Social Psychology, 45, 412-420.

Ingram, R. E., Trenary, L., Odom, M., Berry, L., \& Nelson, T. (2007). Cognitive, affective, and social mechanisms in depression risk: Cognition, hostility, and coping style. Cognition and Emotion, 21, 78-94.

Ingram, R. E., \& Wisnicki, K. (1999). Situational specificity of self-focused attention in dysphoric states. Cognitive Therapy and Research, 23, 625636. 
Keltner, D., Ellsworth, P. C., \& Edwards, K. (1993). Beyond simple pessimism: Effects of sadness and anger on social perception. Journal of Personality and Social Psychology, 5, 740-752.

Kendall, P. C., Hollon, S. D, Beck, A. T, Hammen, C. L., \& Ingram, R. E. (1987). Issues and recommendations regarding use of the Beck Depression Inventory. Cognitive Therapy and Research, 11, 289-299.

Kendall, P. C., \& Watson, D. (Eds.). (1989). Anxiety and depression: Distinctive and overlapping features. San Diego: Academic Press.

Kline, R. B. (2005) Principles and Practices of Structural Equation Modeling (2 ${ }^{\text {nd }}$ ed.). New York: Guilford Press.

Koster, W. H. W., De Raedt, R., Goeleven, E., Franck, E., \& Crombez, G. (2005). Mood-congruent attentional bias in dysphoria: Maintained attention to and impaired disengagement from negative information. Emotion, 5, 446-455.

Koukopoulos, A., Albert, M. J., Sani, G., Koukopoulos, A. E., \& Girardi, P. (2005). Mixed depressive states: Nosologic and therapeutic issues. International Review of Psychiatry, 17, 21-37.

Kuiper, N. A., \& MacDonald, M. R. (1982). Self and other perception in mild depressives. Social Cognition, 1, 223-239.

Little, T. D., Cunningham, W. A., Shahar, G., \& Widaman, K. F. 2002. To parcel or not to parcel: Exploring the question and weighing the merits. Structural Equation Modeling, 9, 151-173. 
MacCallum, R. C., Zhang, S., Preacher, K. J., \& Rucker, D. D. (2002). On the practice of dichotomization of quantitative variables. Psychological Methods, 7, 19-40.

Miller, T. Q., Smith, T. W, Turner, C. W., Guijarro, M. L., \& Hallet, A. J. (1996). A meta-analytic review of research on hostility and physical health. Psychological Bulletin, 119, 322-348.

Moreno, J. K., Fuhriman, A., \& Selby, M. J. (1993). Measurement of hostility, anger, and depression in depressed and nondepressed subjects. Journal of Personality Assessment, 61, 511-523.

Neisser, U. (1967). Cognitive Psychology. East Norwalk, CT: Appleton-CenturyCroft.

Newton, T. L., \& Keicolt-Glaser, J. K. (1995). Hostility and erosion of marital quality during early marriage. Journal of Behavioral Medicine, 18, 601619.

Osman, A., Downs, W. R., Barrios, F. X., Kopper, B. A., Gutierrez, P. M., \& Chiros, C. E. (1997). Factor structure and psychometric characteristics of the Beck Depression Inventory-II. Journal of Psychopathology and Behavioral Assessment, 19, 359-376.

Overall, J. E., \& Hollister, L. E. (1980). Phenomenological classification of depressive disorders. Journal of Clinical Psychology, 36, 372-377.

Overall, J. E., Pull, C., Cabranza, J., \& Cassano, G. (1977). Phenomenological classification of psychiatric patients: Consistency of syndrome 
interpretation by psychiatrists in Italy, France, Mexico and the United States. Journal of Psychiatric Research, 13, 225-236.

Overall, J. E, \& Woodward, J. A. (1975). Conceptual validity of a phenomenological classification of psychiatric patients. Journal of Psychiatric Research, 12, 215-230.

Pasquini, M., Picardi, A., Biondi, M., Gaetano, P., \& Morosini, P. (2004). Relevance of anger and irritability in outpatients with major depressive disorder. Psychopathology, 37, 155-160.

Radloff, L. S. (1977). The CES-D scale: A self-report depression scale for research in the general population. Applied Psychological Measurement, 1, 385-401.

Raja, M., \& Azzoni, A. (2005). Hostility and violence of acute psychiatric inpatients. [Electronic reference] Clinical Practice and Epidemiology in Mental Health, Article 1745-0179-1-11. Retrieved November 8, 2006 from http://www. cpementalhealth.com/content/pdf/1745-0179-1-11.pdf.

Rogers, T. B., Kuiper, N. A., Kirker, W. S. (1977). Self-reference and the encoding of personal information. Journal of Personality and Social Psychology, 35, 677-688.

Rubin, D.B. (1987) Multiple Imputation for Nonresponse in Surveys. New York: J. Wiley \& Sons.

Ruiz, J. M., Smith, T. W., \& Rhodewalt, F. (2001). Distinguishing narcissism and 
hostility: Similarities and differences in interpersonal circumplex and five-factor correlates. Journal of Personality Assessment, 76, 537-555.

Scott, W. D., Ingram, R. E., \& Shadel, W. G. (2003). Sad and hostile moods in dysphoria: Evidence for cognitive specificity in attributions. Journal of Social and Clinical Psychology, 22, 233-252.

Scott, W. D., \& Steidtmann, D. (2006). Dysphoria and hostile cognition: The relationship depends on trait anger. Cognitive Therapy and Research, $30,19-27$.

Sharkin, B. S. (1993). Anger and gender: Theory, research, and implications. Journal of Counseling and Development, 71, 386-389.

Siegle, G. J. (1999) Cognitive and Physiological Aspects of Attention to Personally Relevant Negative Information in Depression. [Electronic reference]. Retrieved June 8, 2009 from http://www.sci.sdsu.edu/CAL/greg/dissert/main/siegle-dissertation.htm.

Siegle, G. J., Granholm, E. Ingram, R. E., \& Matt, G. E. (2001). Pupillary and reaction time measures of sustained processing of negative information in depression. Biological Psychiatry, 49, 624-636.

Siegle, G. J., Ingram, R. E., \& Matt, G. E. (2002). Affective interference: An explanation for negative attention biases in dysphoria. Cognitive Therapy and Research, 26, 73-87.

Siegle, G. J., Steinhauer, S. R., Carter, C. S., Ramel, W., \& Thase, M. E. (2003). Do seconds turn into hours? Relationships between sustained pupil 
dilation in response to emotional information and self-reported rumination. Cognitive Therapy and Research, 27, 365- 382.

Siegle, G. J., Steinhauer, S. R., \& Thase, M. E. (2004). Pupillary assessment and computational modeling of the Stroop task in depression. International Journal of Psychophysiology, 52, 63-76.

Siegler, I. C., Costa, P. T., Brummett, B. H., Helms, M. J., Barefoot, J. C., \& Williams, R. B., et al. (2003). Patterns of change in hostility from college to midlife in the UNC Alumni Heart Study predict high-risk status. Psychosomatic Medicine, 65, 738-745.

Smeets, R., Otgaar, H., Candel, I., \& Wolf, O. T. (2008). True or false? Memory is differentially affected by stress-induced cortisol elevations and sympathetic activity at consolidation and retrieval. Psychoneuroendocrinology, 33, 1378-1386.

Smith, T. W. (1994). In A. W. Siegman, \& T. W. Smith (Eds.), Anger, Hostility, and the Heart (pp.23-42). Hillsdale, NJ: Lawrence Erlbaum Associates.

Smith, T. W., Glazer, K., Ruiz, R. M., \& Gallo, L. C. (2004). Hostility, anger, aggressiveness, and coronary heart disease: An interpersonal perspective on personality, emotion, and health. Journal of Personality, $72,1217-1270$.

Smith, T. W., \& MacKenzie, J. (2006). Personality and risk of physical illness. Annual Review of Clinical Psychology, 2, 435-467.

Smith, T. W., Pope, M. K., Sanders, J. K., Allred, K. D., \& O'Keefe, J. L. (1988). 
Cynical hostility at home and work: Psychosocial vulnerability across domains. Journal of Research in Personality, 22, 525-548.

Snyder, C. R., Crowson, J. J. Jr., Houston, B. K., Kurylo, M., \& Poirier, J. (1997). Assessing automatic hostile thoughts: Development and validation of the HAT scale. Cognitive Therapy and Research, 21, 477-492

Tennen, H., \& Affleck, G. (1990). Blaming others for threatening events. Psychological Bulletin, 108, 209-232.

Vranceanu, A., Gallo, L. C., \& Bogart, L. M. (2006). Hostility and perceptions of social support in ambiguous situations. Journal of Individual Differences, 27, 108-115.

Watkins, P. C., Martin, C. K., \& Stern, L. D. (2000). Unconscious memory bias in depression: Perceptual and conceptual processes. Journal of Abnormal Psychology, 109, 282-289.

Weissman, M., Fox, K., \& Klerman, G. L. (1973). Hostility and depression associated with suicide attempts. American Journal of Psychiatry, 130, 450-455.

Widmayer, S. A. (n.d.). Schema theory: An introduction. Retrieved November 10, 2006 from http://chd.gse.gmu.edu/immersion/knowledgebase/ strategies/cognitivism/SchemaTheory.htm.

Winokur, G. (1985). The validity of neurotic-reactive depression: New data and reappraisal. Archives of General Psychiatry, 42, 116-1122. 
Table 1

Adjectives Used in the Stimulus Task

Hostile Adjectives

Friendly Adjectives

\begin{tabular}{|c|c|c|c|}
\hline Group 1 & Group2 & Group 1 & Group 2 \\
\hline conniving & aggressive & admirable & compassionate \\
\hline crooked & backstabbing & caring & considerate \\
\hline deceitful & cheating & courteous & dependable \\
\hline dishonest & cruel & giving & generous \\
\hline insensitive & greedy & moral & good \\
\hline mean & hurtful & nice & helpful \\
\hline ruthless & lying & reliable & kind \\
\hline selfish & manipulative & responsible & loyal \\
\hline underhanded & sneaky & sincere & respectable \\
\hline unfair & untrustworthy & thoughtful & truthful \\
\hline \multicolumn{2}{|c|}{ Depressive (Sad) Adjectives } & \multicolumn{2}{|c|}{ Nondepressive Adjectives } \\
\hline Group 1 & Group2 & Group 1 & Group 2 \\
\hline down & low & confident & calm \\
\hline sad & miserable & sturdy & strong \\
\hline bleak & lonely & contented & fine \\
\hline unwanted & gloomy & gracious & jovial \\
\hline hurt & heartsick & peaceful & safe \\
\hline rejected & alone & alright & steady \\
\hline sunk & glum & pleasant & sociable \\
\hline sluggish & blue & rational & secure \\
\hline dismal & useless & free & neighborly \\
\hline disinterested & unhappy & relaxed & capable \\
\hline
\end{tabular}


Table 2

Observed Indicator Means and Standard Deviations

Independent Variables and Covariate

HATS total score CES-D-S total score

BDI-II total score

$\begin{array}{lrrr}\text { Mean } & 60.33 & 7.25 & 18.99 \\ \text { SD } & 21.18 & 4.08 & 6.26\end{array}$

Dependent Variables

HSRT HSMax HSSlope HSMem FSRT FSMax FSSlope FSMem

$\begin{array}{lcccccccc}\text { Mean } & 2.43 & 0.63 & 0.04 & 0.97 & 1.77 & 0.56 & 0.03 & 1.47 \\ \text { SD } & 0.89 & 0.26 & 0.03 & 1.06 & 0.68 & 0.22 & 0.02 & 1.16\end{array}$

DSRT DSMax DSSlope DSMem FLRT FLMax FLSlope FLMem

$\begin{array}{lllllllll}\text { Mean } & 2.27 & 0.58 & 0.03 & 1.05 & 1.64 & 0.57 & 0.03 & 1.22\end{array}$

$\begin{array}{lllllllll}\text { SD } & 0.76 & 0.24 & 0.03 & 1.11 & 0.62 & 0.23 & 0.02 & 1.07\end{array}$

FDRT FDMax FDSlope FDMem HDRT HDMax HDSlope HDMem

$\begin{array}{lllllllll}\text { Mean } & 2.51 & 0.63 & 0.04 & 0.97 & 1.97 & 0.64 & 0.04 & 1.30\end{array}$

$\begin{array}{lllllllll}\text { SD } & 1.03 & 0.30 & 0.03 & 1.06 & 0.68 & 0.23 & 0.03 & 0.99\end{array}$

DDRT DDMax DDSlope DDMem

\begin{tabular}{lllll} 
Mean & 2.35 & 0.60 & 0.04 & 0.49 \\
SD & 0.80 & 0.25 & 0.03 & 0.70 \\
\hline
\end{tabular}

Note: $S D=$ standard deviation. Each dependent variable (model indicator) name has a two letter suffix that identifies both the valence and person it represents: HS = self-relevant hostile; $F S=$ self-relevant friendly; $D S=$ self-relevant depressive; 
$L F=$ liked other-relevant friendly; $L D=$ liked other-relevant depressive; $D H=$ disliked other-relevant hostile; $D D=$ disliked other-relevant depressive.

Furthermore each variable name has a suffix that identifies the type of attention measure: $R T=$ reaction time in seconds; Slope = extent of negative slope in the pupil waveform from 3 second after stimulus presentation through 12 seconds after; Max = peak pupil dilation; Mem = number of words from this category recalled at the end of the session. 
Table 3

Indicator Variances and Covariances

HATpar1 HATpar2 HATpar3 CESsad1 CESsad2 CESsad3

$\begin{array}{lrrrrrr}\text { HATpar1 } & 56.927 & & & & & \\ \text { HATpar2 } & 53.684 & 62.661 & & & & \\ \text { HATpar3 } & 43.097 & 42.627 & 47.597 & & & \\ \text { CESsad1 } & 2.272 & 2.214 & 2.184 & 3.172 & & \\ \text { CESsad2 } & 0.037 & -0.067 & 0.034 & 1.360 & 1.921 & \\ \text { CESsad3 } & 1.184 & 0.743 & 1.581 & 1.595 & 1.451 & 2.687 \\ \text { HSRTsec } & 0.537 & 0.694 & 0.515 & -0.117 & -0.047 & -0.146 \\ \text { HSMax } & 1.606 & 1.791 & 1.985 & 0.135 & 0.152 & 0.182 \\ \text { HSSlp } & -0.716 & -0.152 & -0.501 & -0.238 & -0.159 & -0.123 \\ \text { FSRTsec } & -0.079 & 0.073 & 0.125 & -0.091 & -0.012 & -0.036 \\ \text { FSMax } & 0.876 & 0.641 & 1.363 & 0.572 & 0.382 & 0.581 \\ \text { FSSlp } & 2.531 & 2.543 & 1.661 & 0.148 & 0.303 & 0.698 \\ \text { DSRTsec } & 0.357 & 0.331 & 0.301 & 0.059 & 0.079 & 0.109 \\ \text { DSMax } & 2.031 & 1.661 & 1.991 & 0.074 & -0.181 & 0.019 \\ \text { DSSlp } & 2.742 & 2.993 & 3.382 & 0.158 & 0.342 & 0.930 \\ \text { LFRTsec } & -0.057 & 0.259 & -0.015 & -0.141 & -0.030 & -0.042 \\ \text { LFMax } & 0.408 & -0.493 & 1.053 & -0.127 & 0.437 & 0.444 \\ \text { LFSlp } & 1.699 & 0.624 & 1.413 & 0.700 & 0.471 & 0.936 \\ \text { LDRTsec } & 1.035 & 1.248 & 1.090 & 0.303 & 0.046 & 0.214 \\ \text { LDMax } & -0.490 & 0.960 & 2.089 & 0.040 & -0.204 & -0.146 \\ \text { LDSlp } & -1.825 & 1.092 & -0.775 & 0.782 & 0.583 & 0.266 \\ \text { DHRTsec } & 0.452 & 0.757 & 0.267 & 0.091 & 0.015 & 0.033 \\ \text { DHMax } & 2.873 & 2.003 & 2.236 & 0.434 & 0.321 & 0.478 \\ \text { DHSlp } & 4.847 & 4.455 & 3.927 & 0.200 & 0.323 & 0.259 \\ \text { DDRTsec } & 0.584 & 0.665 & 0.418 & -0.161 & 0.021 & -0.061 \\ \text { DDMax } & 4.047 & 3.668 & 1.811 & 0.279 & -0.205 & -0.447 \\ \text { DDSlp } & 6.497 & 7.773 & 4.721 & 0.512 & 0.003 & 0.230 \\ \text { SelfHost } & -1.034 & -0.955 & -0.848 & -0.117 & -0.092 & -0.126 \\ \text { SelfFrnd } & -0.242 & -0.365 & 0.126 & 0.045 & 0.060 & 0.031 \\ \text { SelfSad } & -0.618 & -0.142 & -1.010 & 0.235 & 0.009 & 0.059 \\ \text { LkdFrnd } & -0.408 & -0.352 & -0.133 & 0.185 & 0.258 & 0.258 \\ \text { LkdSad } & -0.388 & -0.283 & -0.178 & 0.167 & 0.160 & 0.122 \\ \text { DslkHost } & -0.486 & -0.334 & -0.233 & -0.071 & -0.101 & -0.129 \\ \text { DslkSad } & 0.406 & 0.458 & 0.122 & -0.088 & -0.016 & -0.023 \\ \text { BDIT2 } & 5.366 & 5.628 & 1.438 & 5.883 & 3.783 & 3.990 \\ \text { Gender } & -0.742 & -0.759 & -0.697 & -0.122 & 0.109 & 0.050\end{array}$


HSRTsec HSMax HSSlp FSRTsec FSMax FSSlp

$\begin{array}{lcrrrrr}\text { HSRTsec } & 0.714 & & & & & \\ \text { HSMax } & 0.058 & 5.514 & & & & \\ \text { HSSlp } & 0.247 & 2.147 & 6.335 & & & \\ \text { FSRTsec } & 0.352 & 0.194 & 0.209 & 0.424 & & \\ \text { FSMax } & -0.160 & 2.690 & 1.436 & -0.247 & 3.852 & \\ \text { FSSlp } & 0.127 & 1.899 & 2.125 & 0.104 & 1.834 & 5.261 \\ \text { DSRTsec } & 0.395 & 0.148 & 0.250 & 0.286 & -0.168 & 0.046 \\ \text { DSMax } & -0.165 & 2.491 & 1.745 & -0.084 & 2.351 & 2.024 \\ \text { DSSlp } & -0.038 & 1.877 & 1.857 & -0.047 & 2.028 & 1.510 \\ \text { LFRTsec } & 0.311 & 0.183 & 0.385 & 0.268 & -0.089 & 0.202 \\ \text { LFMax } & 0.029 & 2.449 & 1.317 & -0.100 & 2.822 & 1.648 \\ \text { LFSlp } & -0.064 & 2.590 & 2.375 & -0.168 & 2.472 & 2.933 \\ \text { LDRTsec } & 0.367 & -0.062 & 0.299 & 0.350 & -0.419 & 0.269 \\ \text { LDMax } & 0.133 & 3.328 & 2.213 & -0.003 & 2.909 & 1.896 \\ \text { LDSlp } & -0.224 & 0.971 & -0.002 & -0.177 & 1.568 & 0.272 \\ \text { DHRTsec } & 0.316 & 0.138 & 0.264 & 0.248 & -0.125 & 0.001 \\ \text { DHMax } & 0.132 & 2.584 & 1.471 & -0.061 & 2.921 & 2.134 \\ \text { DHSlp } & -0.045 & 2.311 & 2.037 & -0.230 & 1.998 & 3.054 \\ \text { DDRTsec } & 0.449 & 0.012 & 0.181 & 0.307 & -0.292 & -0.080 \\ \text { DDMax } & -0.017 & 3.005 & 2.381 & -0.152 & 2.982 & 1.095 \\ \text { DDSlp } & 0.016 & 2.762 & 2.470 & 0.139 & 2.228 & 3.487 \\ \text { SelfHost } & 0.000 & -0.264 & -0.078 & 0.033 & -0.340 & -0.291 \\ \text { SelfFrnd } & -0.043 & -0.126 & -0.127 & 0.066 & -0.249 & 0.015 \\ \text { SelfSad } & 0.007 & -0.560 & -0.234 & 0.012 & -0.082 & -0.127 \\ \text { LkdFrnd } & -0.270 & -0.121 & 0.046 & -0.085 & 0.199 & -0.102 \\ \text { LkdSad } & -0.051 & -0.408 & -0.230 & -0.089 & -0.211 & -0.288 \\ \text { DslkHost } & -0.119 & -0.309 & 0.171 & -0.144 & 0.042 & 0.009 \\ \text { DslkSad } & -0.050 & -0.028 & -0.121 & -0.012 & 0.022 & 0.006 \\ \text { BDIT2 } & 0.422 & 0.801 & -0.012 & 0.306 & 0.795 & -0.278 \\ \text { Gender } & 0.051 & 0.236 & 0.117 & 0.085 & 0.068 & 0.110 \\ & & & & & & \end{array}$

DSRTsec DSMax DSSlp LFRTsec LFMax LFSlp

$\begin{array}{lrrrrrr}\text { DSRTsec } & 0.537 & & & & & \\ \text { DSMax } & -0.298 & 4.706 & & & & \\ \text { DSSlp } & -0.077 & 2.064 & 6.979 & & & \\ \text { LFRTsec } & 0.257 & 0.016 & -0.022 & 0.370 & & \\ \text { LFMax } & -0.034 & 2.014 & 1.749 & 0.016 & 4.403 & \\ \text { LFSlp } & -0.044 & 2.091 & 2.023 & -0.113 & 2.588 & 5.063 \\ \text { LDRTsec } & 0.342 & 0.051 & 0.014 & 0.261 & -0.275 & -0.048\end{array}$


DSRTsec DSMax DSSlp LFRTsec LFMax LFSlp

\begin{tabular}{lcrrrrr} 
LDMax & -0.255 & 2.696 & 2.126 & 0.167 & 3.305 & 2.656 \\
LDSlp & -0.508 & 0.784 & 1.209 & -0.076 & 0.640 & 0.289 \\
DHRTsec & 0.259 & 0.036 & -0.042 & 0.265 & -0.008 & -0.002 \\
DHMax & 0.001 & 2.184 & 2.205 & 0.086 & 3.251 & 2.308 \\
DHSlp & -0.240 & 2.250 & 3.114 & 0.009 & 1.652 & 2.654 \\
DDRTsec & 0.374 & -0.171 & -0.121 & 0.312 & -0.044 & -0.150 \\
DDMax & -0.129 & 3.081 & 2.070 & 0.042 & 2.250 & 1.811 \\
DDSlp & 0.115 & 3.168 & 3.984 & 0.347 & 2.106 & 2.519 \\
SelfHost & -0.019 & -0.222 & -0.413 & 0.020 & -0.543 & -0.234 \\
SelfFrnd & 0.075 & -0.307 & -0.315 & 0.070 & -0.263 & -0.439 \\
SelfSad & 0.024 & -0.257 & -0.405 & 0.001 & -0.140 & -0.348 \\
LkdFrnd & -0.162 & 0.258 & -0.116 & -0.096 & 0.050 & -0.204 \\
LkdSad & 0.031 & -0.382 & -0.311 & -0.054 & -0.178 & -0.243 \\
DslkHost & -0.036 & 0.004 & -0.625 & -0.017 & 0.111 & 0.000 \\
DslkSad & 0.011 & 0.097 & 0.061 & -0.009 & 0.065 & -0.161 \\
BDIT2 & 0.901 & 1.454 & 0.630 & 0.654 & 0.524 & 0.958 \\
Gender & 0.061 & 0.101 & -0.046 & 0.075 & 0.235 & 0.147 \\
& & & & & & \\
& LDRTsec & LDMax & LDSlp & DHRTsec & DHMax & DHSlp \\
\hline
\end{tabular}

$\begin{array}{lcrrrrr}\text { LDRTsec } & 0.956 & & & & & \\ \text { LDMax } & 0.086 & 7.183 & & & & \\ \text { LDSlp } & -0.445 & 1.747 & 7.966 & & & \\ \text { DHRTsec } & 0.281 & 0.138 & 0.020 & 0.428 & & \\ \text { DHMax } & -0.065 & 2.262 & 1.242 & -0.067 & 5.481 & \\ \text { DHSlp } & -0.144 & 2.125 & 1.158 & -0.206 & 3.168 & 7.087 \\ \text { DDRTsec } & 0.340 & -0.032 & -0.213 & 0.353 & -0.130 & -0.454 \\ \text { DDMax } & -0.407 & 2.042 & 1.349 & 0.010 & 3.395 & 3.181 \\ \text { DDSlp } & 0.426 & 3.137 & 0.866 & 0.209 & 3.216 & 4.900 \\ \text { SelfHost } & 0.012 & -0.350 & 0.213 & 0.009 & -0.697 & -0.341 \\ \text { SelfFrnd } & 0.137 & -0.305 & 0.005 & -0.043 & -0.038 & -0.116 \\ \text { SelfSad } & 0.080 & -0.059 & 0.042 & -0.007 & -0.441 & -0.554 \\ \text { LkdFrnd } & -0.093 & 0.017 & 0.057 & -0.144 & 0.042 & 0.076 \\ \text { LkdSad } & -0.027 & -0.369 & -0.026 & -0.073 & -0.334 & -0.333 \\ \text { DslkHost } & -0.005 & 0.081 & -0.007 & -0.019 & -0.020 & -0.046 \\ \text { DslkSad } & -0.032 & 0.220 & -0.034 & -0.019 & -0.078 & -0.074 \\ \text { BDIT2 } & 1.158 & 0.562 & 2.137 & 1.126 & 0.188 & -0.001 \\ \text { Gender } & 0.005 & 0.098 & 0.137 & 0.058 & 0.101 & 0.080\end{array}$




\section{DDRTsec DDMax DDSlp SelfHost SelfFrnd SelfSad}

$\begin{array}{lrrrrrr}\text { DDRTsec } & 0.873 & & & & & \\ \text { DDMax } & -0.461 & 7.237 & & & & \\ \text { DDSlp } & -0.275 & 4.497 & 11.173 & & & \\ \text { SelfHost } & -0.050 & -0.498 & -0.377 & 1.115 & & \\ \text { SelfFrnd } & -0.032 & -0.386 & -0.068 & 0.233 & 1.332 & \\ \text { SelfSad } & -0.031 & -0.402 & -0.399 & 0.327 & 0.056 & 1.228 \\ \text { LkdFrnd } & -0.184 & 0.156 & -0.052 & 0.123 & 0.145 & 0.029 \\ \text { LkdSad } & 0.011 & -0.518 & -0.575 & 0.080 & 0.188 & 0.166 \\ \text { DslkHost } & -0.028 & -0.228 & -0.221 & 0.125 & 0.062 & 0.031 \\ \text { DslkSad } & 0.004 & 0.027 & 0.266 & -0.058 & 0.010 & 0.109 \\ \text { BDIT2 } & 0.866 & 0.976 & 2.150 & -0.474 & -0.234 & 0.305 \\ \text { Gender } & 0.053 & 0.059 & 0.042 & 0.034 & 0.104 & -0.046\end{array}$

LkdFrnd LkdSad DslkHost DslkSad BDIT2 Gender

$\begin{array}{lrrrrrr}\text { LkdFrnd } & 1.134 & & & & & \\ \text { LkdSad } & 0.172 & 0.517 & & & & \\ \text { DslkHost } & 0.089 & 0.097 & 0.967 & & & \\ \text { DslkSad } & -0.022 & 0.048 & -0.018 & 0.480 & & \\ \text { BDIT2 } & 0.732 & 0.349 & -0.248 & -0.369 & 38.992 & \\ \text { Gender } & 0.013 & -0.030 & 0.056 & 0.004 & -0.064 & 0.239\end{array}$

Note: HatParl = first parceled indicator loading on the Hostility construct, HatPar2 = the second loading on this construct, and HatPar3 = the third and final parceled indicator on the Hostility construct. CESsad1 = first parceled indicator loading on the Sadness construct, CESsad2 = the second loading on this construct, and CESsad3 = the third and final parceled indicator on the Sadness construct. Each dependent variable (model indicator) name has a two letter suffix that identifies both the valence and person it represents: HS = selfrelevant hostile; $F S=$ self-relevant friendly; $D S=$ self-relevant depressive; $L F=$ liked other-relevant friendly; $L D=$ liked other-relevant depressive; $D H=$ disliked other-relevant hostile; $D D=$ disliked other-relevant depressive. Furthermore each variable name has a suffix that identifies the type of attention measure: $R T=$ reaction time in seconds; Slope = extent of negative slope in the pupil waveform from 3 second after stimulus presentation through 12 seconds after; Max = peak 
pupil dilation; Indicators with no such prefix or suffix (e.g., LdkFrnd) represent the memory indicators. These indicator names are such that the prefixes Self, Lkd, and Dslk indicate self-, liked other-, and disliked other-relevance, respectively. The suffixes of these indicator names represent valence such that Frnd, Host, and Sad indicate information that is friendly, hostile, and depressive in valence, respectively. BDIT2 $=B D I-I I$ score at the second, in-session administration. Gender was coded such that $1=$ male and $2=$ female. 
Table 4

Loadings, Residuals, and $R^{2}$ Values for Each Indicator

$\underline{\text { Hostility Parcels }} \quad \underline{\text { Sadness Parcels }}$

$\begin{array}{llllll}\text { Loading (SE) } & \text { Loading }^{a} & R^{2} & \text { Loading }(S E) & \text { Loading }^{a} & R^{2}\end{array}$

$\begin{array}{lllllll}\text { Parcel } 1 & 7.41^{* *}(0.20) & 0.98 & 0.96 & 1.21 * *(0.06) & 0.68 & 0.46\end{array}$

$\begin{array}{lllllll}\text { Parcel } 2 & 7.25 * *(0.23) & 0.92 & 0.84 & 1.09 * *(0.05) & 0.78 & 0.62\end{array}$

$\begin{array}{lllllll}\text { Parcel } 3 & 5.82 * *(0.21) & 0.84 & 0.71 & 1.34 * *(0.06) & 0.82 & 0.67\end{array}$

Loading on Person-Related Construct Loading on Emotion-Related

Construct(self, liked, or disiked other) (hostile, friendly, or sad depressive) Loading (SE) Loading ${ }^{a} \quad$ Loading (SE) Loading $^{a} \quad$ Total R $^{2}$

$\underline{\text { Hostile Self-Relevant }}$

$\begin{array}{lrrrrr}\text { RT } & -1.72 * *(0.35) & -2.04 & -1.17 * *(0.35) & -1.38 & 0.64 \\ \text { Slope } & 2.14 *(0.94) & 0.85 & 3.22 * *(0.91) & 1.28 & 0.26 \\ \text { Max } & 4.18^{* *}(1.49) & 1.71 & 5.35^{* *}(1.45) & 2.28 & 0.54 \\ \begin{array}{l}\text { Words } \\ \text { Recalled }\end{array} & -0.54 *(0.24) & -0.52 & -0.68^{* *}(0.24) & -0.64 & 0.04\end{array}$

Friendly Self-Relevant

\begin{tabular}{|c|c|c|c|c|c|}
\hline RT & $-0.61 * *(0.10)$ & -0.93 & $-0.10 \quad(0.12)$ & -0.15 & 0.67 \\
\hline Slope & $1.24 * *(0.38)$ & 0.54 & $1.94 * *(0.38)$ & 0.85 & 0.29 \\
\hline Max & $2.28 * *(0.56)$ & 1.16 & $2.59 * *(0.58)$ & 1.32 & 0.69 \\
\hline Words & $-0.26 * *(0.09)$ & -0.23 & $-0.27 * *(0.10)$ & -0.23 & 0.02 \\
\hline
\end{tabular}


Loading on Person-Related Construct Loading on Emotion-Related

Construct(self, liked, or disiked other) (hostile, friendly, or sad depressive)

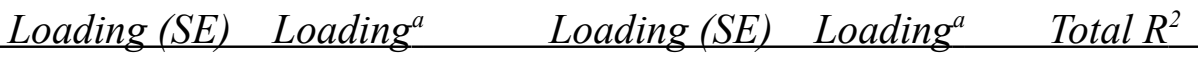

Depressive Self-Relevant

$\begin{array}{lccccc}\text { RT } & -1.20^{* *}(0.22) & -1.63 & -0.74 * *(0.22) & -1.01 & 0.61 \\ \text { Slope } & 2.84 * *(0.89) & 1.07 & 3.53^{* *}(0.85) & 1.34 & 0.26 \\ \text { Max } & 3.51 * *(1.03) & 1.62 & 4.18 * *(1.00) & 1.93 & 0.51 \\ \text { Words } & -0.44 *(0.18) & -0.39 & -0.54 * *(0.17) & -0.48 & 0.03 \\ \text { Recalled } & & & & & \end{array}$

Friendly Liked Other-Relevant

$\begin{array}{lllllll}\text { RT } & -0.48^{* *}(0.09) & -0.79 & 0.03 & (0.11) & 0.04 & 0.68 \\ \text { Slope } & 2.46^{* *}(0.64) & 1.16 & 3.00^{* *}(0.69) & 1.32 & 0.55 \\ \text { Max } & 2.62^{* *}(0.61) & 1.18 & 2.91^{* *}(0.65) & 1.40 & 0.61 \\ \text { Words } & 0.24 * *(0.10) & 0.23 & 0.01 & (0.09) & 0.01 & 0.06 \\ \text { Recalled } & & & & & & \end{array}$

Depressive Liked Other-Relevant

$\begin{array}{llllll}\text { RT } & -1.77 * *(0.38) & -1.81 & -1.28^{* *}(0.37) & -1.31 & 0.39 \\ \text { Slope } & 2.42^{* *}(0.74) & 0.86 & 7.14^{* *}(1.72) & 0.86 & 0.05 \\ \text { Max } & 6.18^{* *}(1.75) & 2.31 & 2.41^{* *}(0.66) & 2.67 & 0.50 \\ \text { Words } & -0.44^{*}(0.18) & -0.62 & -0.61 * *(0.18) & -0.85 & 0.09 \\ \text { Recalled } & & & & & \end{array}$


Loading on Person-Related Construct Loading on Emotion-Related

Construct(self, liked, or disiked other) (hostile, friendly, or sad depressive)

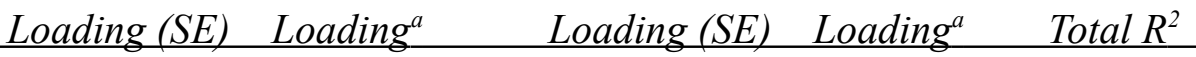

Hostile Disliked Other-Relevant

$\begin{array}{lrrrrrrrr}\text { RT } & -5.45 & (4.40) & -8.33 & -5.00 & (4.40) & -7.65 & 0.61 \\ \text { Slope } & 36.94 & (32.01) & 13.87 & & 37.53 & (32.08) & 14.10 & 0.49 \\ \text { Max } & 34.75 & (30.43) & 14.73 & 35.61 & (30.41) & 15.09 & 0.62 \\ \text { Words } & 0.01 & (0.84) & 0.01 & -0.11 & (0.84) & -0.11 & 0.02 \\ \text { Recalled } & & & & & & & & \end{array}$

Depressive Disliked Other-Relevant

\begin{tabular}{lccccccc} 
RT & $-2.80^{* *}(0.84)$ & -2.99 & $-2.25^{* *}(0.84)$ & -2.41 & 0.51 \\
Slope & $12.12 * *(4.41)$ & 3.63 & $13.28^{* *}(4.39)$ & 3.99 & 0.47 \\
Max & $12.29 * *$ & $(4.33)$ & 4.57 & $12.92 * *(4.31)$ & 4.80 & 0.58 \\
Words & 0.17 & $(0.19)$ & 0.25 & 0.15 & $(0.19)$ & 0.22 & 0.00 \\
$\begin{array}{l}\text { Recalled } \\
\text { R }\end{array}$ & & & & & & \\
\hline
\end{tabular}

${ }^{\mathrm{a} C}$ Common Metric Completely Standardized Solution

Note: Estimates from the measurement model solution. $R T=$ reaction time in seconds; Slope = extent of negative slope in the pupil waveform from 3 second after stimulus presentation through 12 seconds after; Max = peak pupil dilation; Words Recalled $=$ number of words from this category recalled at the end of the session. 
Table 6

Fit Indices

\begin{tabular}{lllllll}
\multicolumn{8}{c}{ Measurement Model } \\
$\chi^{2}$ & $d f$ & $p$ & RMSEA & $\begin{array}{c}\text { RMSEA } \\
90 \% \text { CI }\end{array}$ & NNFI & CFI \\
\hline & & & & & & \\
878.21 & $471<.001$ & 0.067 & $0.058 ; 0.075$ & 0.91 & 0.92 \\
\hline
\end{tabular}

$\underline{\text { Structural Model }}$

\begin{tabular}{lllllll}
$\chi^{2}$ & $d f$ & $p$ & RMSEA & $\begin{array}{l}\text { RMSEA } \\
90 \% \text { CI }\end{array}$ & NNFI & CFI \\
\hline 965.30 & 523 & $<.001$ & 0.066 & $0.058 ; 0.074$ & 0.90 & 0.92 \\
\hline
\end{tabular}

Note: $C I=$ Confidence Interval 
Table 7

Beta Paths from the Structural Model Solution

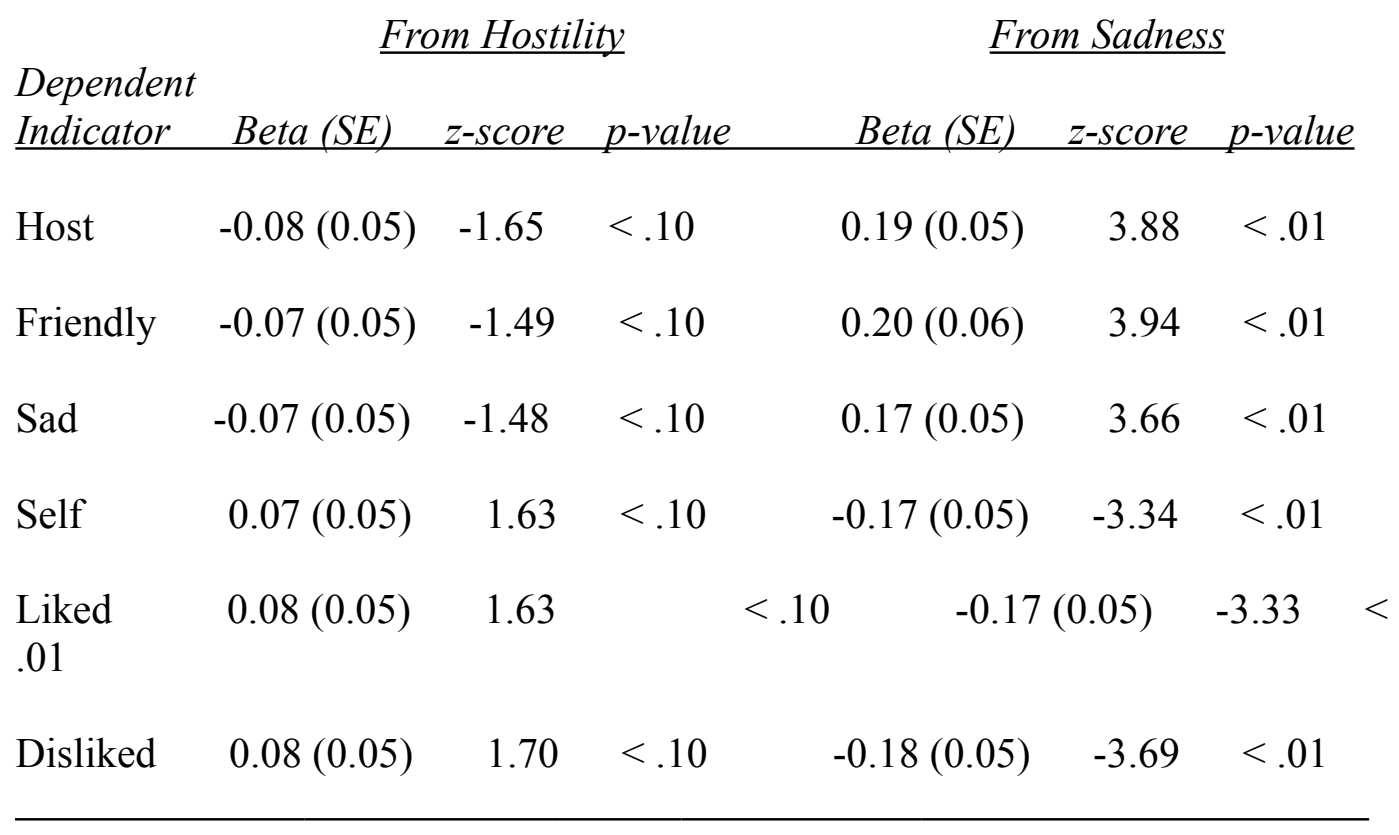

Note: $S E=$ standard error. 
Table 8

Gamma Paths

$\underline{\text { BDI-II }}$

Latent

Construct

Hostility

Sadness

Host

Friendly

Sad

Self

Liked

Disliked
Gamma (SE) p-value

$0.02(0.01)<.01$

$0.12(0.01)<.01$

$-0.06(0.01)<.01$

$-0.06(0.01)<.01$

$-0.06(0.01)<.01$

$0.06(0.01)<.01$

$0.06(0.01)<.01$

$0.05(0.01)<.01$ $\underline{\text { Gender }}$

Gamma (SE) p-value

$-0.44(0.08)<.01$

$0.18(0.09)<.05$

$-0.61(0.08)<.01$

$-0.64(0.09)<.01$

$-0.62(0.08)<.01$

$0.63(0.08)<.01$

$0.63(0.09)<.01$

$0.76(0.08)<.01$

Note: Estimates from the structural model solution. SE = standard error. 


\section{Figure Caption}

Figure 1: Estimates are from the completely standardized solution associated with the structural model. Hostility and Sadness = the independent latent constructs; Host, Friendly, and Sad represent the latent dependent constructs associated with emotion-relevant aspects of attention to information; Self, Liked, and Disliked represent the latent dependent constructs associated with the self-relevant and other-relevant aspects of attention to information. 
Figure 1. Beta paths and construct variances/covariances for the structural model.

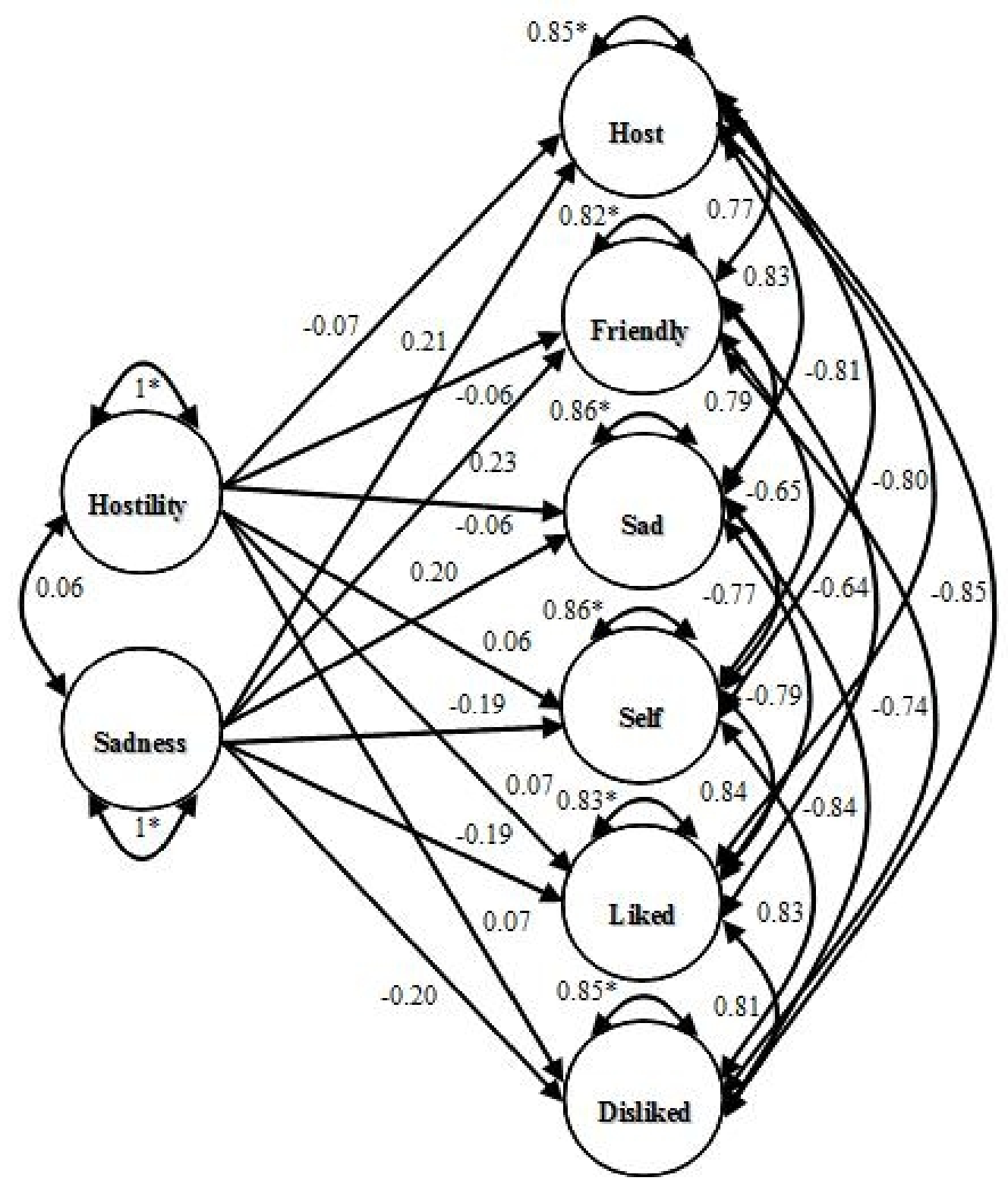




\section{Appendix}

\section{Questionnaires Administered}

Demographic Questionnaire

Participant Number:

Date: $\tau_{\mathrm{mm}}{ }^{\prime} \mathrm{dd}^{\prime} \frac{}{\text { yyyy }}$

Sex (circle one): $\quad$ Male Female

Birthdate: $\overline{\mathrm{mm}}^{\prime} \frac{}{\mathrm{dd}}{ }^{\prime} \frac{}{\text { yyyy }}$

$\begin{array}{lllll}\text { Year in school (circle one): } & \text { Fr } & \text { So } & \mathrm{Jr} & \mathrm{Sr}\end{array}$

Race/Ethnicity:

Do you wear glasses or contacts, or have you ever had you vision corrected? (circle one):

Yes No

If yes, please describe:

Are you currently taking any medications (circle one): $\quad$ Yes No

If yes, please list the medications and dose if you know it: 
Name:

Marital Status:

Age:

Sex:

Occupation: Education:

Instructions: This questionnaire consists of 21 groups of statements. Please read each group of statements carefully, and then pick out the one statement in each group that best describes the way you have been feeling during the past two weeks, including today. Circle the number beside the statement you have picked. If several statements in the group seem to apply equally well, circle the highest number for that group. Be sure that you do not choose more than one statement for any group, including Item 16 (Changes in Sleeping Pattern) or Item 18 (Changes in Appetite).

\author{
1. Sadness \\ $0 \quad$ I do not feel sad. \\ 1 I feel sad much of the time. \\ 2 I am sad all the time. \\ 3 I am so sad or unhappy that I can't stand it.
}

\section{Pessimism}

0 I am not discouraged about my future.

1 I feel more discouraged about my future than I used to be.

2 I do not expect things to work out for me.

3 I feel my future is hopeless and will only get worse.

\section{Past Failure}

0 I do not feel like a failure.

1 I have failed more than I should have.

2 As I look back, I see a lot of failures.

3 I feel I am a total failure as a person.

\section{Loss of Pleasure}

0 I get as much pleasure as I ever did from the things I enjoy.

1 I don't enjoy things as much as I used to.

2 I get very little pleasure from the things I used to enjoy.

3 I can't get any pleasure from the things I used to enjoy.

\section{Guilty Feelings}

0 I don't feel particularly guilty.

1 I feel guilty over many things I have done or should have done.

2 I feel quite guilty most of the time.

3 I feel guilty all of the time.

\section{Punishment Feelings}

0 I don't feel I am being punished.

1 I feel I may be punished.

2 I expect to be punished.

3 I feel I am being punished.

\section{Self-Dislike}

0 I feel the same about myself as ever.

1 I have lost confidence in myself.

2 I am disappointed in myself.

3 I dislike myself.

\section{Self-Criticalness}

0 I don't criticize or blame myself more than usual.

1 I am more critical of myself than I used to be.

2 I criticize myself for all of my faults.

3 I blame myself for everything bad that happens.

\section{Suicidal Thoughts or Wishes}

0 I don't have any thoughts of killing myself.

1 I have thoughts of killing myself, but I would not carry them out.

2 I would like to kill myself.

3 I would kill myself if I had the chance.

\section{Crying}

0 I don't cry anymore than I used to.

1 I cry more than I used to.

2 I cry over every little thing.

3 I feel like crying, but I can't. 


\section{Agitation}

0 I am no more restless or wound up than usual.

1 I feel more restless or wound up than usual.

2 I am so restless or agitated that it's hard to stay still.

3 I am so restless or agitated that I have to keep moving or doing something.

\section{Loss of Interest}

0 I have not lost interest in other people or activities.

1 I am less interested in other people or things than before.

2 I have lost most of my interest in other people or things.

3 It's hard to get interested in anything.

\section{Indecisiveness}

0 I make decisions about as well as ever.

1 I find it more difficult to make decisions than usual.

2 I have much greater difficulty in making decisions than I used to.

3 I have trouble making any decisions.

\section{Worthlessness}

0 I do not feel I am worthless.

1 I don't consider myself as worthwhile and useful as I used to.

2 I feel more worthless as compared to other people.

3 I feel utterly worthless.

\section{Loss of Energy}

0 I have as much energy as ever.

1 I have less energy than I used to have.

2 I don't have enough energy to do very much.

3 I don't have enough energy to do anything.

\section{Changes in Sleeping Pattern}

0 I have not experienced any change in my sleeping pattern.

1a I sleep somewhat more than usual.

1b I sleep somewhat less than usual.

2a I sleep a lot more than usual.

$2 \mathrm{~b}$ I sleep a lot less than usual.

3a I sleep most of the day.

3b I wake up 1-2 hours early and can't get back to sleep.

\section{Irritability}

0 I am no more irritable than usual.

1 I am more irritable than usual.

2 I am much more irritable than usual.

3 I am irritable all the time.

\section{Changes in Appetite}

0 I have not experienced any change in my appetite.

1a My appetite is somewhat less than usual.

1b My appetite is somewhat greater than usual.

2a My appetite is much less than before.

$2 b \quad$ My appetite is much greater than usual.

3a I have no appetite at all.

3b I crave food all the time.

\section{Concentration Difficulty}

0 I can concentrate as well as ever.

1 I can't concentrate as well as usual.

2 It's hard to keep my mind on anything for very long.

3 I find I can't concentrate on anything.

\section{Tiredness or Fatigue}

0 I am no more tired or fatigued than usual.

1 I get more tired or fatigued more easily than usual.

2 I am too tired or fatigued to do a lot of the things I used to do.

3 I am too tired or fatigued to do most of the things I used to do.

\section{Loss of Interest in Sex}

0 I have not noticed any recent change in my interest in sex.

1 I am less interested in sex than I used to be.

2 I am much less interested in sex now.

3 I have lost interest in sex completely. 
Using the 5 point scale shown below, indicate how uncharacteristic or characteristic each of the following statements is in describing you:

$1=$ extremely uncharacteristic of me

$2=$ somewhat uncharacteristic of me

$3=$ neither uncharacteristic nor characteristic of me

$4=$ somewhat characteristic of me

$5=$ extremely characteristic of me

1. Some of my friends think I am a hothead. $\quad \begin{array}{lllllll}1 & 2 & 3 & 4 & 5\end{array}$

2. If I have to resort to violence to protect my $\quad \begin{array}{llllllll}1 & 2 & 3 & 4 & 5\end{array}$ rights, I will.

3. When people are especially nice to me, I wonder $\begin{array}{llllllll}1 & 2 & 3 & 4 & 5\end{array}$ what they want.

4. I tell my friends openly when I disagree with them. $\begin{array}{lllllll}1 & 2 & 3 & 4 & 5\end{array}$

5. I want have become so mad that I have broken $\quad \begin{array}{llllll}1 & 2 & 3 & 4 & 5\end{array}$ things.

6. I can't help getting into arguments when people $\quad \begin{array}{lllllll}1 & 2 & 3 & 4 & 5\end{array}$ disagree with me.

7. I wonder why sometimes I feel so bitter about $\quad \begin{array}{lllllll}1 & 2 & 3 & 4 & 5\end{array}$ things.

8. Once in a while, I can't control the urge to strike $\quad \begin{array}{lllllll}1 & 2 & 3 & 4 & 5\end{array}$ another person.

$\begin{array}{lllllll}\text { 9. I am an even-tempered person. } & 1 & 2 & 3 & 4 & 5\end{array}$

10. I am suspicious of overly-friendly strangers. $\quad \begin{array}{lllllll}1 & 2 & 3 & 4 & 5\end{array}$

11. I have threatened people I know.

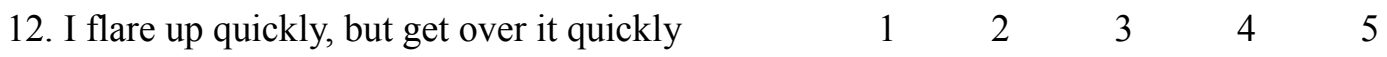

13. Given enough provocation, I may hit another $\quad \begin{array}{lllllll}1 & 2 & 3 & 4 & 5\end{array}$ person. 
14. When people annoy me, I may tell them what I $\quad \begin{array}{llllll}1 & 2 & 3 & 4 & 5\end{array}$ think of them.

15. I am sometimes eaten up with jealousy. $\quad \begin{array}{lllllll}1 & 2 & 3 & 4 & 5\end{array}$

16. I can think of no good reason for ever hitting a $\quad \begin{array}{llllll}1 & 2 & 3 & 4 & 5\end{array}$ person.

17. At times, I feel I have gotten a raw deal out of life. $\begin{array}{lllllll}1 & 2 & 3 & 4 & 5\end{array}$

18. I have trouble controlling my temper. $\quad \begin{array}{lllllll} & 1 & 2 & 3 & 4 & 5\end{array}$

19. When frustrated, I let my irritation show. $\quad \begin{array}{lllllll}1 & 2 & 3 & 4 & 5\end{array}$

20. I sometimes feel that people are laughing at me $\quad \begin{array}{llllll}1 & 2 & 3 & 4 & 5\end{array}$ behind my back.

21. I often find myself disagreeing with people. $\quad \begin{array}{lllllll}1 & 2 & 3 & 4 & 5\end{array}$

22. If somebody hits me, I hit back. $\quad \begin{array}{llllll} & 1 & 2 & 3 & 4 & 5\end{array}$

23. I sometimes feel like a powder keg, ready to $\quad \begin{array}{lllllll}1 & 2 & 3 & 4 & 5\end{array}$ explode.

24. Other people always seem to get the breaks. $\quad \begin{array}{llllll}1 & 2 & 3 & 4 & 5\end{array}$

25. There are people who pushed me so far, that we $\quad \begin{array}{lllllll}1 & 2 & 3 & 4 & 5\end{array}$ came to blows.

26. I know that "friends" talk about me behind $\quad \begin{array}{llllll}1 & 2 & 3 & 4 & 5\end{array}$ my back.

27. My friends say that I am somewhat argumentative. $\begin{array}{lllllll}1 & 2 & 3 & 4 & 5\end{array}$

28. Sometimes I fly off the handle for no good reason. $\begin{array}{lllllll}1 & 2 & 3 & 4 & 5\end{array}$

29. I get into fights a little more than the average $\quad \begin{array}{llllll}1 & 2 & 3 & 4 & 5\end{array}$ person. 


\section{CES-D}

Below is a list of the ways you might have felt or behaved. Please tell me how often you have felt this way during the past week.

\section{During the Past Week}

$0=$ Rarely or none of the time (less than 1 day)

$1=$ Some or a little of the time $(1-2$ days $)$

$2=$ Occasionally or a moderate amount of time ( $3-4$ days $)$

$3=$ Most or all of the time (5 -7 days $)$

1. I was bothered by things that usually $\quad 0 \quad 113$ don't bother me.

2. I did not feel like eating; my appetite $\quad 0 \quad 1 \quad 3$ was poor.

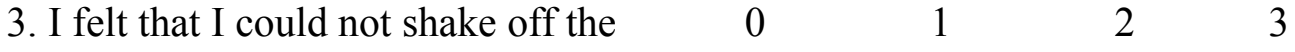
blues even with help from my family or friends.

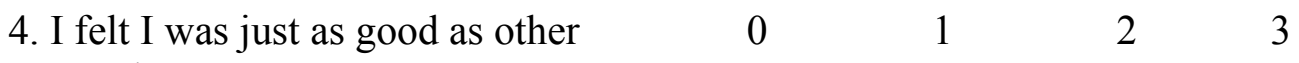
people.

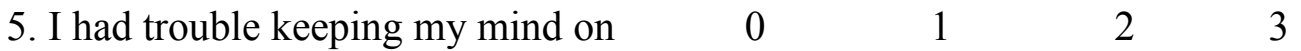
what I was doing.

$\begin{array}{lllll}\text { 6. I felt depressed. } & 0 & 1 & 2 & 3\end{array}$

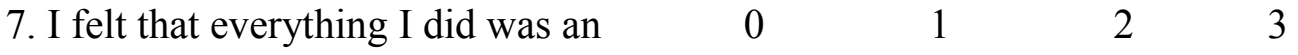
effort.

$\begin{array}{lllll}\text { 8. I felt hopeful about the future. } & 0 & 1 & 2 & 3\end{array}$

9. I thought my life had been a failure. $\quad 0 \quad 0 \quad 1 \quad 3$

$\begin{array}{lllll}\text { 10. I felt fearful. } & 0 & 1 & 2 & 3\end{array}$

$\begin{array}{lllll}\text { 11. My sleep was restless. } & 0 & 1 & 2 & 3\end{array}$

12. I was happy. $\quad 0 \quad 1123$ 
13. I talked less than usual.

0

14. I felt lonely.

15. People were unfriendly.

16. I enjoyed life.

17. I had crying spells.

18. I felt sad.

19. I felt that people dislike me.

20. I could not get "going."
0

0

0

0

0

0

0

\section{1}

2

2

2

3

3

3

3

3

3

\section{HATS}

Take a few minutes to think about your previous week. Read and respond to each of the following items according to the frequency with which that thought (or one similar to it) has occurred in the last week. Use the 5-point scale shown below.

$1=$ not at all $\quad 2=$ sometimes $3=$ moderately often $\quad 4=$ often $\quad 5=$ all the time

1. I hate this person so much I could kill him/her! $\quad \begin{array}{llllll}1 & 2 & 3 & 4 & 5\end{array}$

$\begin{array}{lllllll}\text { 2. I want to kill this person! } & 1 & 2 & 3 & 4 & 5\end{array}$

$\begin{array}{lllllll}\text { 3. I wish this person was dead. } & 1 & 2 & 3 & 4 & 5\end{array}$

4. If I could get away with it, I'd kill this person! $\quad \begin{array}{llllll}1 & 2 & 3 & 4 & 5\end{array}$

$\begin{array}{lllllll}\text { 5. I want to beat the hell out of this person! } & 1 & 2 & 3 & 4 & 5\end{array}$

$\begin{array}{lllllll}\text { 6. I'd like to knock his/her teeth out. } & 1 & 2 & 3 & 4 & 5\end{array}$

7. I can think of a lot of terrible things I'd like to $\quad \begin{array}{llllll}1 & 2 & 3 & 4 & 5\end{array}$ see happen to that person.

$\begin{array}{llllllll}\text { 8. I want to smack this person! } & 1 & 2 & 3 & 4 & 5\end{array}$

$\begin{array}{lllllll}\text { 9. I want to hit this person. } & 1 & 2 & 3 & 4 & 5\end{array}$ 
10. I want to destroy something right now! $\quad \begin{array}{lllllll} & 1 & 2 & 3 & 4 & 5\end{array}$

11. If someone really wants to mess with me, then $\quad \begin{array}{llllll}1 & 2 & 3 & 4 & 5\end{array}$ they deserve to get roughed up.

12. What an idiot! $\quad \begin{array}{llllll} & 1 & 2 & 3 & 4 & 5\end{array}$

$\begin{array}{llllll}\text { 13. This person is a loser. } & 1 & 2 & 3 & 4 & 5\end{array}$

$\begin{array}{llllllll}\text { 14. I hate stupid people. } & 1 & 2 & 3 & 4 & 5\end{array}$

$\begin{array}{llllllll}\text { 15. What a jerk! } & 1 & 2 & 3 & 4 & 5\end{array}$

16. She is so annoying. $\quad \begin{array}{llllll}1 & 2 & 3 & 4 & 5\end{array}$

$\begin{array}{lllllll}\text { 17. What the hell is this person doing? } & 1 & 2 & 3 & 4 & 5\end{array}$

18. I think this person is rude. $\quad \begin{array}{llllll}1 & 2 & 3 & 4 & 5\end{array}$

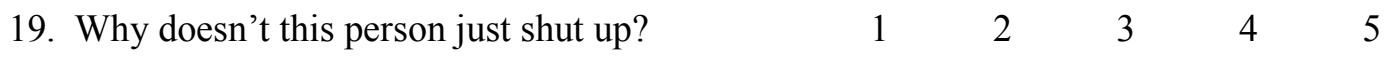

20. I wish they would just shut up and go away. $\quad \begin{array}{lllllll}1 & 2 & 3 & 4 & 5\end{array}$

21. This person makes me feel angry. $\quad \begin{array}{lllllll} & 1 & 2 & 3 & 4 & 5\end{array}$

$\begin{array}{llllllll}\text { 22. I have to get this person back. } & 1 & 2 & 3 & 4 & 5\end{array}$

$\begin{array}{llllllll}\text { 23. I want to get back at this person. } & 1 & 2 & 3 & 4 & 5\end{array}$

24. I just want to hurt this person as bad as s/he hurt $\quad \begin{array}{lllllll}1 & 2 & 3 & 4 & 5\end{array}$ me.

25. I want to get revenge. $\quad \begin{array}{lllllll}1 & 2 & 3 & 4 & 5\end{array}$

26. I want to treat this person like s/he treated me. $\quad \begin{array}{lllllll}1 & 2 & 3 & 4 & 5\end{array}$

27. I'll show this person! $\quad 1 \quad \begin{array}{llllll} & 2 & 3 & 4 & 5\end{array}$

28. I should do something to this person. $\quad \begin{array}{lllllll}1 & 2 & 3 & 4 & 5\end{array}$

29. When someone attacks me like this person did, $\quad \begin{array}{lllllll}1 & 2 & 3 & 4 & 5\end{array}$ I attack them back.

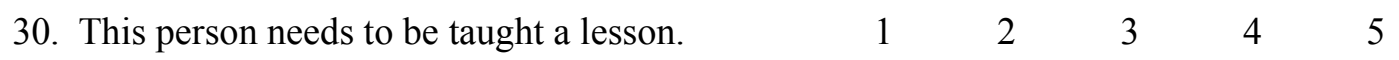




\section{Incidental Recall Prompt}

Please think back to the longer computer task and write down all of the words that you can remember below. You will have 3 full minutes. 